\title{
Analysis on general meshes of a discrete duality finite volume method for subsurface flow problems
}

\author{
A. Njifenjou • H. Donfack • I. Moukouop-Nguena
}

Received: 24 March 2012 / Accepted: 17 December 2012 / Published online: 30 January 2013

(C) The Author(s) 2013. This article is published with open access at Springerlink.com

\begin{abstract}
This work presents and analyzes, on unstructured grids, a discrete duality finite volume method (DDFV method for short) for 2D-flow problems in nonhomogeneous anisotropic porous media. The derivation of a symmetric discrete problem is established. The existence and uniqueness of a solution to this discrete problem are shown via the positive definiteness of its associated matrix. Properties of this matrix combined with adequate assumptions on data allow to define a discrete energy norm. Stability and error estimate results are proven with respect to this norm. $L^{2}$-error estimates follow from a discrete Poincaré inequality and an $L^{\infty}$-error estimate is given for a $P_{1}$ DDFV solution. Numerical tests and comparison with other schemes (especially those from FVCA5 benchmark) are provided.
\end{abstract}

\footnotetext{
A. Njifenjou $(\varangle) \cdot$ I. Moukouop-Nguena

National Advanced School of Engineering,

University of Yaounde I, P.O. Box: 8390,

Yaounde, Cameroon

e-mail: njifa2000@yahoo.fr

I. Moukouop Nguena

e-mail:imoukouo@yahoo.com

\begin{abstract}
A. Njifenjou
African Institute of Computer Science,

P.O. Box: 2263, Libreville, Gabon
\end{abstract}

\section{H. Donfack}

Faculty of Science, University of Yaounde I,

P.O. Box: 812, Yaounde, Cameroon

e-mail: hubertdonfack@yahoo.fr
}

Keywords Flow problems • Nonhomogeneous anisotropic media $\cdot$ Discrete duality finite volumes • Stability and error estimates $\cdot$ Numerical tests

Mathematics Subject Classifications (2010) 35J65 • $65 \mathrm{~N} 15 \cdot 74 \mathrm{~S} 10$

\section{Introduction and the model problem}

Efficient schemes are required for addressing flow problems in geologically complex media. The most important criteria of efficiency are (1) mass conservation in grid blocks, (2) accurate approximation of Darcy velocity, (3) capability for dealing with anisotropic flow on unstructured grids and diverse heterogeneities (relevant to absolute permeability, porosity, etc.), and (4) easy implementation. The following schemes are well known for meeting many of the previous efficiency criteria: (1) mixed finite element methods (see, for instance, [7, 36]), (2) control-volume finite element methods (see, for instance, [10, 38]), (3) mimetic finite difference methods (see, for instance, $[6,8]$ and references therein), (4) cell-centered finite volume methods (see, for instance, [14-18] and certain references therein; see also $[5,24,25])$, (5) multipoint flux approximation (with pioneer works from [2,37]; see also $[1,11]$ and some contributions to convergence analysis of MPFA O-scheme like [23]), and (6) discrete duality finite volume methods (DDFV methods for short). The DDFV methods come in two formulations. The first formulation to appear is based on interface flux computations for primary and dual meshes, accounting with the interface flux continuity. The pioneer works for this formulation 
are [31] and [21] followed by [20, 27, 30, 32] and [19]. The second formulation of DDFV is based on pressure gradient reconstructions over a diamond grid. This formulation has been first introduced in [13] (see also [33, 34]). Note that this second formulation has been given focused attention by some mathematicians as Andreianov, Boyer, and Hubert who have greatly contributed to its mathematical development. Indeed, key ideas involved in the pressure gradient reconstruction approach have been generalized by these authors (see [3]) to nonlinear operators of Leray-Lions type. Motivated by the possibility of increasing the order of convergence of the pressure gradient reconstruction method for nonlinear operators, Boyer and Hubert have proposed in [9] the so-called modified DDFV. In what follows, the first formulation of DDFV is named flux-based DDFV and the second one is called gradient-based DDFV. Note that flux is understood here in the sense of outward normal component of the Darcy velocity on cell boundaries.

As shown in FVCA5 benchmark test problems, the flux-based DDFV and gradient-based DDFV may lead to very different orders of convergence for some diffusion models. In fact, these two formulations describe two different ways for solving flow problems in the framework of DDFV. The theoretical analysis strategy of the flux-based DDFV developed in $[30,32]$ on a rectangular mesh follows closely ideas from [14]. Very different ingredients are used in [3] and [9] for getting either the numerical scheme or the error estimates.

AS pointed out by some authors (namely Moukouop-Nguena and Njifenjou in FVCA5 benchmark [22]), there exist many variants for the flux-based DDFV. Following the ideas exposed in [14], some authors (see [30]) have, on a square grid, investigated the stability and convergence properties of a variant of the flux-based DDFV, named reused-value method. This work is a continuation of the one in [30]. In other words, our purpose in this paper was to look for sufficient conditions on general grids for proving the stability and the convergence of the reused-value method. More precisely, the main points of our work are the following:

1. A theoretical analysis of the flux-based DDFV on general grids for flow problems in polygonal (possibly nonconvex) anisotropic nonhomogeneous media

2. Numerical validations on general meshes, thanks to some FVCA5 benchmark problems exposed in Section 4

3. Comparison of our flux-based DDFV with other numerical schemes (including gradient-based DDFV methods) in terms of convergence rate of approximate pressure and pressure gradient for $L^{2}$-norm.

Let us emphasize that one novelty of this work may be exhibited in terms of methodology for formulating and theoretically analyzing a DDFV model on general grids in two dimensions. We start with a $2 \mathrm{D}$ linear diffusion problem: Find a real function $\varphi$ defined over $\bar{\Omega}$ such that

$-\operatorname{div}(K \operatorname{grad} \varphi)=f \quad$ in $\Omega$

$\varphi=0 \quad$ on $\quad \Gamma$

where $\Omega$ is a given open polygonal domain and $\Gamma$ its boundary and where $f$ is a given function. $K=K(x)$, with $x=\left(x_{1}, x_{2}\right)^{t} \in \Omega$, is a full matrix which meets the following conditions (symmetry, boundedness, and uniform ellipticity):

$\forall 1 \leq i, j \leq 2, \quad K_{i j}(x)=K_{j i}(x) \quad$ a.e. in $\Omega$

$$
\text { and } K_{i j} \in L^{\infty}(\Omega)
$$

$\forall \xi \in \mathbb{R}^{2} \quad \gamma_{\min }|\xi|^{2} \leq \xi^{T} K(x) \xi \leq \gamma_{\max }|\xi|^{2} \quad$ a.e. in $\Omega$

where $\gamma_{\min }$ and $\gamma_{\max }$ are strictly positive real numbers independent of $x,|$.$| denotes the euclidian norm in \mathbb{R}^{2}$, and $K_{i j}($.$) is the component of K$.

Remark 1.1 Note that the flux-based DDFV analyzed here applies to 3D diffusion phenomena. We should come back to this point in further developments.

The elliptic operator involved in the left-hand side of Eq. (1.1) is similar to the one arising in subsurface single- or multiphase flows (in petroleum and hydrogeology engineering), and this motivates our study.

\section{The discrete problem: existence and uniqueness}

We start with exhibiting the discrete problem. Then, we focus on existence and uniqueness of a discrete solution.

\subsection{Domain discretizations: some definitions and notations}

For the polygonal (not necessarily convex) domain $\Omega$, we consider matching unstructured primary meshes $\mathcal{P}$ made up of arbitrary convex polygons called primary cells (see Fig. 1). Nevertheless, the method applies to nonmatching meshes as numerically demonstrated in 


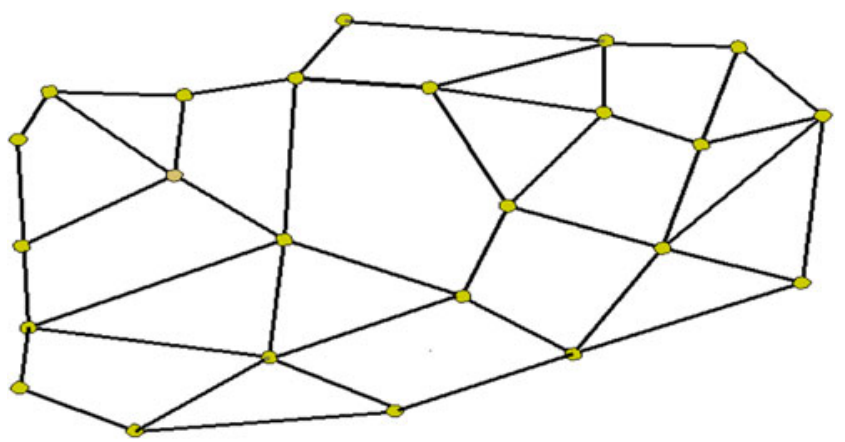

Fig. 1 Example of a primary matching unstructured mesh

Section 4. Let us introduce some definitions very useful for our presentation.

Definition 2.1 An edgepoint is any point (from $\bar{\Omega}$, the closure of $\Omega$ in the sense of the usual topology of $\mathbb{R}^{2}$ ) located over an edge from $\mathcal{P}$ and different from the extremities of that edge.

Definition 2.2 Two edgepoints $I$ and $J$ are called neighboring edgepoints if they share some vertex $V$ in the sense that $I$ and $J$ belong to two different edges intersecting in $V$.

In the context of unstructured primary meshes, the definition of a discrete energy norm similar to the one in [30] requires that the cellpoints lie inside certain perimeters to be defined. For this reason, the main steps for defining the cellpoints are as follows: (1) Choose arbitrarily one point (different from a vertex) on each edge of the mesh $\mathcal{P}$. This process generates a finite family of edgepoints, denoted by $\mathcal{E}$. (2) Join every pair of neighboring edgepoints by a dotted straight line. By this way, we generate an auxiliary mesh denoted by $\mathcal{A}$. (3) Fix arbitrarily one point inside any auxiliary cell completely embedded in a primary cell. By doing so, one generates a finite family of cellpoints that may be identified with the set $\mathcal{P}$ of primary cells. Figure 2 illustrates the location of edgepoints and cellpoints.

Remark 2.3 Note that in 3D, for a given primary mesh on $\Omega$, the associated auxiliary mesh is generated easily. Indeed, since each primary cell $C$ involves a finite number of faces, there is the same number of face points lying on the boundary of $C$. Therefore, one could associate with these face points the smallest polyhedron containing all of them.

According to the variational theory of linear elliptic problems, the system (1.1)-(1.2) possesses a unique solution $\varphi$ in the so-called Sobolev space $H_{0}^{1}(\Omega)$, un- der the assumptions (1.3)-(1.4) and the condition that $f \in L^{2}(\Omega)$. Let us assume that the diffusion matrix coefficient $K$ is a piecewise constant matrix function over $\Omega$. This assumption is realistic for engineering problems as reservoir or aquifer simulations.

Definition 2.4 A mesh $\mathcal{M}$ defined over $\Omega$ is compatible with the discontinuities of $K$ in $\Omega$ if these discontinuities are confined in the interfaces of $\mathcal{M}$.

Main assumptions We assume that the primary mesh $\mathcal{P}$ is compatible with the discontinuities of $K$ in $\Omega$ and that these discontinuities divide $\Omega$ into a finite number of convex subdomains $\left\{\Omega_{s}\right\}_{s \in S}$. On the other hand, we suppose that the restriction over $\Omega_{s}$ of the exact solution to Eqs. (1.1)-(1.2), denoted by $\varphi_{\left.\right|_{\Omega_{s}}}$, satisfies to

$\varphi_{\left.\right|_{\Omega_{s}}} \in C^{2}\left(\bar{\Omega}_{s}\right) \quad \forall s \in S$.

\subsection{Formulation of the discrete problem}

Let us now focus on investigating the flux-based DDFV formulation of Eqs. (1.1)-(1.2). The investigated DDFV scheme should be a linear system involving the quantities $\left\{u_{P}\right\}_{P \in \mathcal{P}}$ and $\left\{u_{P^{*}}\right\}_{P^{*} \in \mathcal{D}}$ as discrete unknowns, where $\mathcal{D}$ represents the dual mesh (to be defined later). These unknowns are expected to be reasonable approximations of $\left\{\varphi_{P}\right\}_{P \in \mathcal{P}}$ (cellpoint pressures) and $\left\{\varphi_{P^{*}}\right\}_{P^{*} \in \mathcal{D}}$ (internal vertex pressures), respectively, where $\varphi_{P}=\varphi\left(x_{1}^{P}, x_{2}^{P}\right)$ and $\varphi_{P^{*}}=$ $\varphi\left(x_{1}^{P^{*}}, x_{2}^{P^{*}}\right)$.

\subsubsection{Molecules for DDFV computation of fluxes}

Let $C_{P}$ be a primary cell, where $P$ is the corresponding cellpoint. We integrate the two sides of the balance Eq. (1.1) in $C_{P}$. Applying the Ostrogradski's theorem to the integral in the left-hand side of this equation leads to computing the flux across the bound-

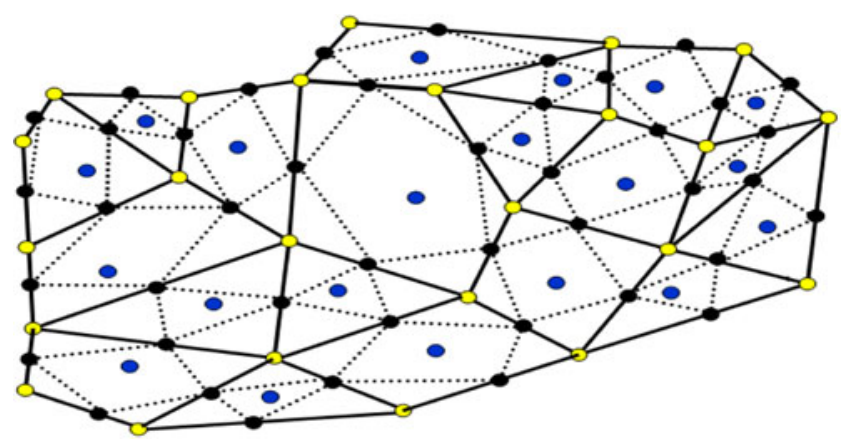

Fig. 2 A primary mesh (full lines) and the associated auxiliary mesh (dotted lines), including edgepoints and cellpoints in black and blue colors, respectively 
ary of $C_{P}$. Using a suitable quadrature formula for approximating this flux, a discrete balance equation is derived. For illustrating our ideas, we consider an edge $\left[A^{*} B^{*}\right]$ associated with the primary cell $C_{P}$ (see Fig. 3). Let $K^{P}$ be the absolute permeability tensor of the cell $C_{P}$. Denoting by $\xi_{\left[A^{*} B^{*}\right]}^{P}$ the unit normal vector to $\left[A^{*} B^{*}\right]$ exterior to $C_{P}$, the flux expression over the edge $\left[A^{*} B^{*}\right]$ viewed as part of the boundary of $C_{P}$ is given by

$$
\begin{aligned}
Q_{\left[A^{*} B^{*}\right]}^{P}= & -\int_{\left[A^{*} I\right]} \operatorname{grad} \varphi \cdot\left(K^{P} \xi_{\left[A^{*} B^{*}\right]}^{P}\right) d \gamma \\
& -\int_{\left[I B^{*}\right]} \operatorname{grad} \varphi \cdot\left(K^{P} \xi_{\left[A^{*} B^{*}\right]}^{P}\right) d \gamma .
\end{aligned}
$$

Before starting with the flux computations across the subedges $\left[A^{*} I\right]$ and $\left[I B^{*}\right]$, let us set

$\overrightarrow{P I}=|\overrightarrow{P I}| \sigma_{P}, \quad \overrightarrow{A^{*} B^{*}}=\left|\overrightarrow{A^{*} B^{*}}\right| \tau_{h}$

where $h=\max \{\operatorname{size}(\mathcal{P}), \operatorname{size}(\mathcal{D})\}$.

Then, it is easily seen that the following identity holds:

$K^{P} \xi_{\left[A^{*} B^{*}\right]}^{P}=a_{h}\left(K^{P}\right) \sigma_{P}-b_{h}\left(K^{P}\right) \tau_{h}$

where the real numbers $a_{h}\left(K^{P}\right)$ and $b_{h}\left(K^{P}\right)$ are given by the relations

$$
\begin{aligned}
& a_{h}\left(K^{P}\right)=\frac{1}{\cos \theta_{h}^{P, I}}\left(\xi_{\left[A^{*} B^{*}\right]}^{P}\right)^{t} K^{P} \xi_{\left[A^{*} B^{*}\right]}^{P}, \\
& b_{h}\left(K^{P}\right)=\frac{1}{\cos \theta_{h}^{P, I}}\left(\xi_{[P I]}^{B^{*}}\right)^{t} K^{P} \xi_{\left[A^{*} B^{*}\right]}^{P}
\end{aligned}
$$

where $\theta_{h}^{P, I}$ is the angle defined by the vectors $\sigma_{P}$ and $\xi_{\left[A^{*} B^{*}\right]}^{P}$, and where $\xi_{[P I]}^{B^{*}}$ denotes the unit normal vector to $[P I]$ exterior to the triangle $\left(P I B^{*}\right)$. Note that $0 \leq$ $\theta_{h}^{P, I}<\frac{\pi}{2}$, and therefore, $0<\cos \theta_{h}^{P, I} \leq 1$.

\subsubsection{Flux computations across the primary grid edges}

The decomposition of $K^{P} \xi_{\left[A^{*} B^{*}\right]}^{P}$ given by Eq. (2.4) lets the first integral in the right-hand side of Eq. (2.2) be expressed as

$$
\begin{aligned}
-\int_{\left[I A^{*}\right]} & \operatorname{grad} \varphi \cdot\left(K^{P} \xi_{\left[A^{*} B^{*}\right]}^{P}\right) d \gamma \\
= & b_{h}\left(K^{P}\right)\left[\varphi_{I}-\varphi_{A^{*}}\right]+a_{h}\left(K^{P}\right) \frac{h_{I A^{*}}}{h_{P I}} \\
& \times\left[\varphi_{p}-\varphi_{I}\right]+T_{\left[I A^{*}\right]}^{P}
\end{aligned}
$$

where $h_{I A^{*}}=\left|\overrightarrow{I A^{*}}\right|, h_{P I}=|\overrightarrow{P I}|$ and where the truncation error $T_{\left[I A^{*}\right]}^{P}$ is given by

$$
\begin{aligned}
T_{\left[I A^{*}\right]}^{P}=-\frac{a_{h}\left(K^{P}\right)}{2}[ & h_{I A^{*}}^{2}\left(\sigma_{P}\right)^{t} \varphi^{\prime \prime}(M) \tau_{h} \\
& \left.-h_{I A^{*}} h_{P I}\left(\sigma_{P}\right)^{t} \varphi^{\prime \prime}(Q) \sigma_{P}\right]
\end{aligned}
$$

with $M \in\left[I A^{*}\right], Q \in[P I]$, and $\varphi^{\prime \prime}($.$) being the Hessian$ of $\varphi$. Note that $\varphi^{\prime \prime}($.) exists, thanks to Eq. (2.1).

Let us continue with performing the flux computation across $\left[I B^{*}\right]$. For this aim, we use again the decomposition of $\xi_{\left[A^{*} B^{*}\right]}^{P}$, and therefore, it follows that

$$
\begin{aligned}
& -\int_{\left[I B^{*}\right]} \operatorname{grad} \varphi \cdot\left(K^{P} \xi_{\left[A^{*} B^{*}\right]}^{P}\right) d \gamma \\
& =b_{h}\left(K^{P}\right)\left[\varphi_{B^{*}}-\varphi_{I}\right] \\
& \quad+a_{h}\left(K^{P}\right) \frac{h_{I B^{*}}}{h_{P I}}\left[\varphi_{P}-\varphi_{I}\right]+T_{\left[I B^{*}\right]}^{P}
\end{aligned}
$$

where $h_{I B^{*}}=\left|\overrightarrow{I B^{*}}\right|$ and where $T_{\left[I B^{*}\right]}^{P}$ is the truncation error given by

$$
\begin{aligned}
T_{\left[I B^{*}\right]}^{P}=-\frac{a_{h}\left(K^{P}\right)}{2}[ & h_{I B^{*}}^{2}\left(\sigma_{P}\right)^{t} \varphi^{\prime \prime}(N) \tau_{h} \\
& \left.-h_{I B^{*}} h_{P I}\left(\sigma_{P}\right)^{t} \varphi^{\prime \prime}(Q) \sigma_{P}\right]
\end{aligned}
$$

Fig. 3 Two molecules for a DDFV computation of the flux across the edge $\left[A^{*} B^{*}\right]$. Left: $\left[A^{*} B^{*}\right]$ lies inside $\Omega$, Right: $\left[A^{*} B^{*}\right]$ is part of the boundary of $\Omega$
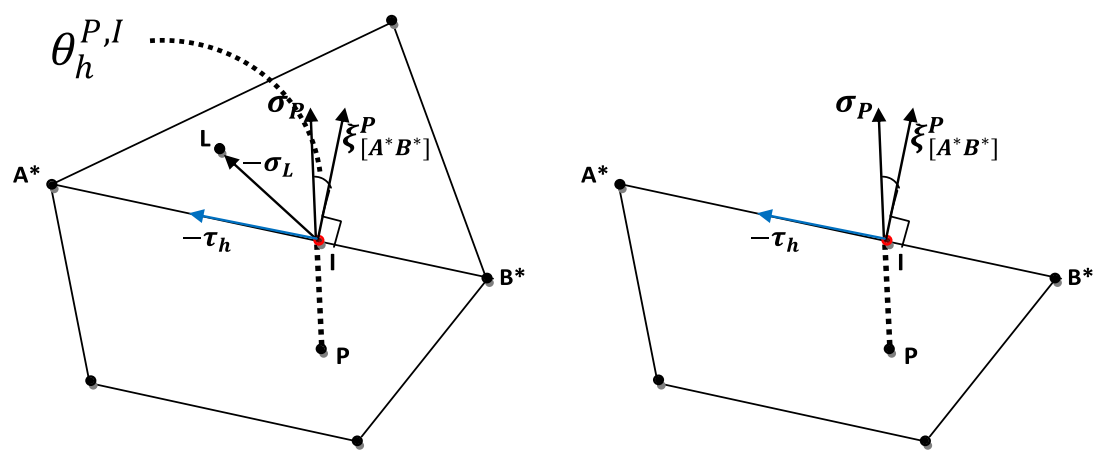
with $N \in\left[I B^{*}\right]$. Thus, the total exact flux across the edge $\left[A^{*} B^{*}\right]$ viewed as part of the boundary of the cell $C_{P}$ reads

$$
\begin{aligned}
Q_{\left[A^{*} B^{*}\right]}^{P}= & b_{h}\left(K^{P}\right)\left[\varphi_{B^{*}}-\varphi_{A^{*}}\right] \\
& +a_{h}\left(K^{P}\right) \frac{h_{A^{*} B^{*}}}{h_{P I}}\left[\varphi_{P}-\varphi_{I}\right]+T_{I\left(A^{*}, B^{*}\right)}^{P}
\end{aligned}
$$

where for a fixed $Q \in[P I]$ and fixed $M, N \in\left[A^{*} B^{*}\right]$, we have set

$$
\begin{aligned}
T_{I\left(A^{*}, B^{*}\right)}^{P}=\frac{a_{h}\left(K^{P}\right)}{2}[ & h_{A^{*} B^{*}} h_{P I}\left(\sigma_{P}\right)^{t} \varphi^{\prime \prime}(Q) \sigma_{P} \\
& -h_{I A^{*}}^{2}\left(\sigma_{P}\right)^{t} \varphi^{\prime \prime}(M) \tau_{h} \\
& \left.-h_{I B^{*}}^{2}\left(\sigma_{P}\right)^{t} \varphi^{\prime \prime}(N) \tau_{h}\right] .
\end{aligned}
$$

For estimating the truncation error $T_{I\left(A^{*}, B^{*}\right)}^{P}$, we start with some useful notations. First of all, recall that $\mathcal{E}$ is the set made of edgepoints (from the primary mesh of course). We denote by $\mathcal{E}^{\text {int }}$ the subset of $\mathcal{E}$ made up of edgepoints lying in $\Omega, \mathcal{E}^{\text {ext }}$ the subset of $\mathcal{E}$ made up of edgepoints lying on the boundary of $\Omega$, and $\mathcal{E}^{P}$ (for $P \in$ $\mathcal{P}$ ) the subset of $\mathcal{E}$ made up of edgepoints lying on the boundary of the primary cell $C_{P}$.

Remark 2.5 Note that the set $\mathcal{E}$ will sometimes be identified with the set of primary edges since there is a trivial bijection between the two sets. In the same order of ideas, the set of cellpoints and the set of vertices will sometimes be identified with the set $\mathcal{P}$ of primary cells and the set $\mathcal{D}$ of dual cells, respectively. At last, primary mesh and primal mesh mean the same thing in this work.

We should also need the following key notion:

Definition 2.6 The system $(\mathcal{P}, \mathcal{E})$ defines a regular mesh system if the following condition is fulfilled: There exists $\theta \in] 0, \frac{\pi}{2}[$, not depending on $h$, such that

$0 \leq \theta_{h}^{P, I} \leq \frac{\pi}{2}-\theta \quad \forall P \in \mathcal{P} \quad \forall I \in \mathcal{E}^{P}$.

- Let us give some examples of regular mesh system in the sense of the previous definition: (1) An obvious example is obtained with $\mathcal{P}$ being the set of square cells and $\mathcal{E}$ the set of edge midpoints. In this example, $\theta=\frac{\pi}{4}$. (2) Another example is got with $\mathcal{P}$ taken to be the set of equilateral triangles and $\mathcal{E}$ the set of edge midpoints. In this example, it is clear that we may take $\theta=\frac{\pi}{6}$.
Ingredients are gathered for estimating truncation errors. In this connection, it is easily seen that the following result holds:

Proposition 2.7 Assume that the mesh system $(\mathcal{P}, \mathcal{E})$ defines a regular mesh system in the sense of the previous definition and that there exists $0<\varpi \leq 1$, mesh independent, such that

$\forall P \in \mathcal{P} \quad \forall I \in \mathcal{E}^{P} \quad \varpi h \leq h_{P I}, h_{A_{I}^{*} B_{I}^{*}} \leq h$

where $A_{I}^{*}, B_{I}^{*} \in \mathcal{D}$ are extremities of the only edge (from the cell $C_{P}$ ) involving the edgepoint $I$. Under the assumptions (1.3) and (2.1), there exists a strictly positive number $C$, mesh independent, such that

$\left|T_{I\left(A^{*}, B^{*}\right)}^{P}\right| \leq C h^{2}$.

The relation (2.14) is a consistency property and so naturally allows to approximate $Q_{\left[A^{*} B^{*}\right]}^{P}$ as it follows

$$
\begin{aligned}
Q_{\left[A^{*} B^{*}\right]}^{P} \approx & b_{h}\left(K^{P}\right)\left[\varphi_{B^{*}}-\varphi_{A^{*}}\right] \\
& +a_{h}\left(K^{P}\right) \frac{h_{A^{*} B^{*}}}{h_{P I}}\left[\varphi_{P}-\varphi_{I}\right] .
\end{aligned}
$$

If $\left[A^{*} B^{*}\right]$ is part of the domain boundary, the edge pressure $\varphi_{I}$ is given by the Dirichlet conditions (notice that in this case, $\varphi_{A^{*}}$ and $\varphi_{B^{*}}$ are also given as $A^{*}$ and $B^{*}$ should be lying on the boundary). Otherwise, [ $\left.A^{*} B^{*}\right]$ is an interface between the cell $C_{P}$ and some primary cell denoted by $C_{L}$; in this context, the edge pressure $\varphi_{I}$ is unknown. But, thanks to the principle of flux continuity, one can approximate it with a linear function of $\varphi_{P}, \varphi_{L}, \varphi_{A^{*}}$, and $\varphi_{B^{*}}$. For investigating the above-mentioned linear function, we compute the flux across $\left[A^{*} B^{*}\right]$ viewed as part of the boundary of $C_{L}$. For this purpose, we set

$K^{L} \xi_{\left[A^{*} B^{*}\right]}^{L}=\widehat{a}_{h}\left(K^{L}\right) \sigma_{L}+\widehat{b}_{h}\left(K^{L}\right) \tau_{h}$

where the real numbers $\widehat{a}_{h}\left(K^{L}\right)$ and $\widehat{b}_{h}\left(K^{L}\right)$ are given by the relations

$\widehat{a}_{h}\left(K^{L}\right)=\frac{1}{\cos \theta_{h}^{L, I}}\left(\xi_{\left[A^{*} B^{*}\right]}^{P}\right)^{t} K^{L} \xi_{\left[A^{*} B^{*}\right]}^{P}$,

$\widehat{b}_{h}\left(K^{L}\right)=\frac{1}{\cos \theta_{h}^{L, I}}\left(\xi_{[I L]}^{B^{*}}\right)^{t} K^{L} \xi_{\left[A^{*} B^{*}\right]}^{P}$

where $\theta_{h}^{L, I}$ is the angle defined by the vectors $-\sigma_{L}$ and $\xi_{\left[A^{*} B^{*}\right]}^{P}$ and where $\xi_{[I L]}^{B^{*}}$ denotes the unit normal vector to $[I L]$ exterior to the half-plane of $\mathbb{R}^{2}$ containing the point $B^{*}$ and being bordered by the straight line $(I L)$. Performing the flux computation over the inter- 
element $\left[A^{*} B^{*}\right]$, viewed as part of the boundary of $C_{L}$ (see Fig. 3), leads to

$$
\begin{aligned}
Q_{\left[A^{*} B^{*}\right]}^{L}= & \widehat{b}_{h}\left(K^{L}\right)\left[\varphi_{A^{*}}-\varphi_{B^{*}}\right] \\
& +\widehat{a}_{h}\left(K^{L}\right) \frac{h_{A^{*} B^{*}}}{h_{I L}}\left[\varphi_{L}-\varphi_{I}\right]+T_{\left[A^{*} B^{*}\right]}^{L}
\end{aligned}
$$

where for a fixed $Q^{\prime} \in[I L]$ and fixed $M^{\prime}, N^{\prime} \in$ $\left[A^{*} B^{*}\right]$, we have set

$$
\begin{aligned}
T_{I\left(A^{*}, B^{*}\right)}^{L}=\frac{\widehat{a}_{h}\left(K^{L}\right)}{2}[ & h_{A^{*} B^{*}} h_{I L}\left(\sigma_{L}\right)^{t} \varphi^{\prime \prime}\left(Q^{\prime}\right) \sigma_{L} \\
& -h_{I A^{*}}^{2}\left(\sigma_{L}\right)^{t} \varphi^{\prime \prime}\left(M^{\prime}\right) \tau_{h} \\
& \left.-h_{I B^{*}}^{2}\left(\sigma_{L}\right)^{t} \varphi^{\prime \prime}\left(N^{\prime}\right) \tau_{h}\right] .
\end{aligned}
$$

Thus, this flux can be approximated with the expression

$$
\begin{aligned}
Q_{\left[A^{*} B^{*}\right]}^{L} \approx & \widehat{b}_{h}\left(K^{L}\right)\left[\varphi_{A^{*}}-\varphi_{B^{*}}\right] \\
& +\widehat{a}_{h}\left(K^{L}\right) \frac{h_{A^{*} B^{*}}}{h_{I L}}\left[\varphi_{L}-\varphi_{I}\right] .
\end{aligned}
$$

The approximate fluxes $Q_{\left[A^{*} B^{*}\right]}^{P}$ and $Q_{\left[A^{*} B^{*}\right]}^{L}$ meet the principle of flux continuity over the interface between $C_{P}$ and $C_{L}$ if and only if the approximate edgepoint pressure $\varphi_{I}$ satisfies to the following relation:

$$
\begin{aligned}
\varphi_{I}= & \frac{1}{\left[a_{h}\left(K^{P}\right) \frac{h_{A^{*} B^{*}}}{h_{P I}}+\widehat{a}_{h}\left(K^{L}\right) \frac{h_{A^{*} B^{*}}}{h_{I L}}\right]} \\
& \times\left\{\left[b_{h}\left(K^{P}\right)-\widehat{b}_{h}\left(K^{L}\right)\right]\left[\varphi_{B^{*}}-\varphi_{A^{*}}\right]\right. \\
& \left.+a_{h}\left(K^{P}\right) \frac{h_{A^{*} B^{*}}}{h_{P I}} \varphi_{p}+\widehat{a}_{h}\left(K^{L}\right) \frac{h_{A^{*} B^{*}}}{h_{I L}} \varphi_{L}\right\} .
\end{aligned}
$$

This is a consistent approximation for $\varphi_{I}$ in the sense that the corresponding truncation error vanishes when $h$ goes to zero (thanks to Proposition 2.7). So, replacing $\varphi_{I}$ in Eq. 2.15 by its approximate value yields the following conservative scheme:

$$
\begin{aligned}
Q_{\left[A^{*} B^{*}\right]}^{P} \approx & {\left[\frac{a_{h}\left(K^{P}\right) \widehat{a}_{h}\left(K^{L}\right) h_{A^{*} B^{*}}}{a_{h}\left(K^{P}\right) h_{I L}+\widehat{a}_{h}\left(K^{L}\right) h_{P I}}\right]\left[\varphi_{P}-\varphi_{L}\right] } \\
& +\left[\frac{\widehat{a}_{h}\left(K^{L}\right) b_{h}\left(K^{P}\right) h_{P I}+a_{h}\left(K^{P}\right) \widehat{b}_{h}\left(K^{L}\right) h_{I L}}{\widehat{a}_{h}\left(K^{L}\right) h_{P I}+a_{h}\left(K^{P}\right) h_{I L}}\right] \\
& \times\left[\varphi_{B^{*}}-\varphi_{A^{*}}\right] .
\end{aligned}
$$

\subsubsection{DDFV flux balance equations in primary cells}

Using the previous notations and thanks to the consistency of the previous DDFV flux approximations (see Proposition 2.7), the approximate flux balance within $C_{P}$ is

$$
\begin{aligned}
& \sum_{I \in \mathcal{E}^{P} \cap \mathcal{E}^{\text {int }}}\left[\frac{a_{h}\left(K^{P}\right) \widehat{a}_{h}\left(K^{L}\right) h_{A^{*} B^{*}}}{a_{h}\left(K^{P}\right) h_{I L}+\widehat{a}_{h}\left(K^{L}\right) h_{P I}}\left(\varphi_{P}-\varphi_{L}\right)\right. \\
& \left.+\frac{\widehat{a}_{h}\left(K^{L}\right) b_{h}\left(K^{P}\right) h_{P I}+a_{h}\left(K^{P}\right) \widehat{b}_{h}\left(K^{L}\right) h_{I L}}{\widehat{a}_{h}\left(K^{L}\right) h_{P I}+a_{h}\left(K^{P}\right) h_{I L}}\left(\varphi_{B^{*}}-\varphi_{A^{*}}\right)\right] \\
& +\sum_{I \in \mathcal{E}^{P} \cap \mathcal{E}^{\mathrm{ext}}} a_{h}\left(K^{P}\right) \frac{h_{A^{*} B^{*}}}{h_{P I}} \varphi_{P} \approx \int_{C_{P}} f(x) d x \quad \forall P \in \mathcal{P}
\end{aligned}
$$

with

$\varphi_{E^{*}}=0 \quad \forall E^{*} \in \mathcal{D} \cap \partial \Omega$

where $A^{*}$ and $B^{*}$ are extremities of the edge containing $I$ and lying on the boundary of the primary cell $C_{P}$ and where $L \in \mathcal{P}$ is such that $\overline{C_{P}} \cap \overline{C_{L}}=\left[A^{*} B^{*}\right]$.

\subsubsection{Necessity of defining a dual grid}

It is clear that the number of discrete unknowns $\left\{\varphi_{P}\right\}_{P \in \mathcal{P}}$ and $\left\{\varphi_{P^{*}}\right\}_{P^{*} \in \mathcal{D}}$ is greater than the number of equations in the system (2.23)-(2.24). For closing this system, we look for discrete equations corresponding to flux balance over dual cells. For this purpose, we define a dual mesh as described in Fig. 4. For carrying out our technique, we need to introduce the notion of pseudoedge associated with dual cells. This notion, illustrated in Fig. 5, is defined as

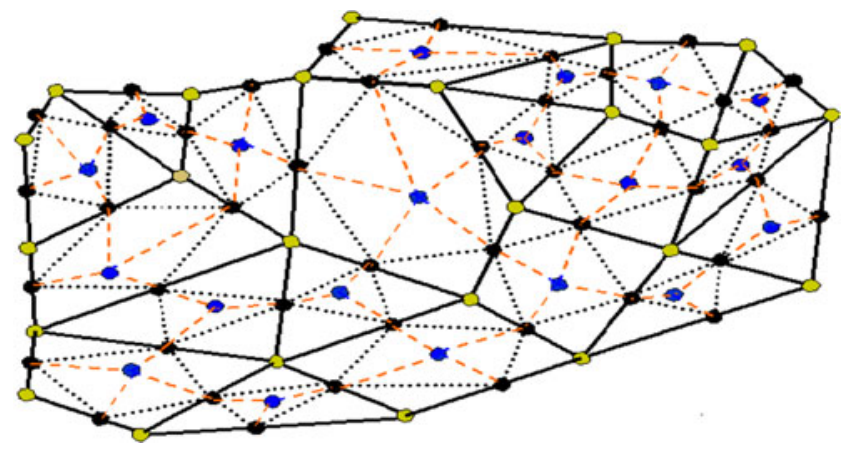

Fig. 4 Combination of a primary mesh (full lines) and the associated dual mesh (red discontinuous lines) including the auxiliary mesh (black dotted lines) 


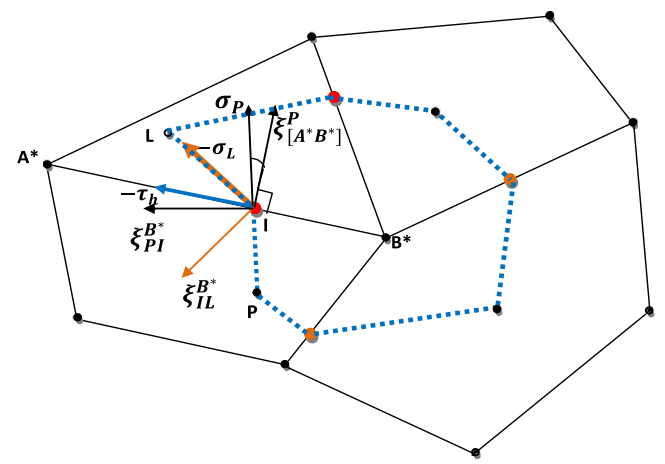

Fig. 5 A dual cell (blue discontinuous line) with its four pseudoedges that intersect primal edges at red points from $\mathcal{E}$

Definition 2.8 Let $P$ and $L$ be two cellpoints from the primary mesh (i.e., $P, L \in \mathcal{P}$ ) such that the corresponding primary cells $C_{P}$ and $C_{L}$ are adjacent, and consider $I \in \mathcal{E}^{P} \cap \mathcal{E}^{L}$ (recall that $\mathcal{E}^{E}$, for $E \in \mathcal{P}$, is the set of edgepoints from $\mathcal{E}$ lying on the boundary of the cell $C_{E}$ ). The line $[P I] \cup[I L]$ defines a pseudoedge, denoted by $[P I L]$, whose extremities are $P$ and $L$.

Remark 2.9 Note that in our presentation, $I$ is in general an angular point of [ $P I L]$. To our knowledge, only the particular case corresponding to $[P I L]$ defined as a straight line has been theoretically investigated so far (see [9] for instance).

We will say that a pseudo-edge is associated with a dual cell $C_{A^{*}}$ if it is part of the boundary of $C_{A^{*}}$.

Remark 2.10 Note that the boundary of any dual cell is a union of a finite number of pseudo-edges (see Fig. 5).

\subsubsection{DDFV flux balance equations in dual cells}

Let us now look for discrete balance equations over dual cells. This is carried out through the following steps:

Step 1: Local integration of mass balance equation

We start with integrating the two sides of Eq. (1.1) in a dual cell $C_{B^{*}}$ represented in Fig. 5. Applying the Ostrogradski's theorem and exploiting Remark 2.10 leads to

$$
\sum_{I \in \mathcal{E}^{B^{*}}}-\int_{[P I L]} K \operatorname{grad} \varphi \cdot n_{B^{*}} d \gamma=\int_{C_{B^{*}}} f(x) d x
$$

where $n_{B^{*}}$ stands for the outward unit normal vector to the boundary of $C_{B^{*}}$ and where $[P I L]$ is a pseudoedge associated with the dual cell $C_{B^{*}}$. Recall that $\mathcal{E}^{B^{*}}$ is the set of edgepoints lying in the boundary of the dual cell $C_{B^{*}}$.

Step 2: Flux computations and truncation errors

Let us look for a flux approximation across the pseudoedge $[P I L]$ viewed as part of the boundary of $C_{B^{*}}$. Denoting by $Q_{[P I L]}^{B^{*}}$ the exact flux over [PIL], it can be expressed by the relation

$$
\begin{aligned}
Q_{[P I L]}^{B^{*}}= & -\int_{[P I]} \operatorname{grad} \varphi \cdot\left(K^{P} \xi_{[P I]}^{B^{*}}\right) d \gamma \\
& -\int_{[I L]} \operatorname{grad} \varphi \cdot\left(K^{L} \xi_{[I L]}^{B^{*}}\right) d \gamma .
\end{aligned}
$$

We should first focus on computations of the flux across $[P I]$. So, define the following decomposition of $K^{P} \xi_{[P I]}^{B^{*}}$ :

$K^{P} \xi_{[P I]}^{B^{*}}=c_{h}\left(K^{P}\right) \sigma_{P}-d_{h}\left(K^{P}\right) \tau_{h}$

where $\sigma_{P}$ and $\tau_{h}$ are unit vectors defined by Eq. (2.3). It is clear that

$c_{h}\left(K^{P}\right)=b_{h}\left(K^{P}\right)$

$d_{h}\left(K^{P}\right)=\frac{1}{\cos \theta_{h}^{P, I}}\left(\xi_{[P I]}^{B^{*}}\right)^{t} K^{P} \xi_{[P I]}^{B^{*}}>0$

(recall that $\theta_{h}^{P, I}$ denotes the angle defined by the vectors $\sigma_{P}$ and $\left.\xi_{\left[A^{*} B^{*}\right]}^{P}\right)$. Therefore,

$$
\begin{aligned}
& -\int_{[P I]} \operatorname{grad} \varphi\left(K^{P} \xi_{[P I]}^{B^{*}}\right) d \gamma \\
& =-b_{h}\left(K^{P}\right) \int_{[P I]} \operatorname{grad} \varphi \cdot \sigma_{P}+d_{h}\left(K^{P}\right) \int_{[P I]} \operatorname{grad} \varphi \cdot \tau_{h} \\
& =b_{h}\left(K^{P}\right)\left(\varphi_{P}-\varphi_{I}\right)+d_{h}\left(K^{P}\right) \frac{h_{P I}}{h_{A^{*} B^{*}}}\left(\varphi_{B^{*}}-\varphi_{A^{*}}\right) \\
& \quad+T_{[P I], A^{*}}^{B^{*}}
\end{aligned}
$$

where

$$
\begin{aligned}
T_{[P I], A^{*}}^{B^{*}}=\frac{d_{h}\left(K^{P}\right)}{2}( & h_{P I}^{2}\left(\tau_{h}\right)^{t} \varphi^{\prime \prime}\left(Q_{P I}\right) \sigma_{P} \\
& +\frac{h_{P I} h_{I B^{*}}^{2}}{h_{A^{*} B^{*}}}\left(\tau_{h}\right)^{t} \varphi^{\prime \prime}\left(Q_{I B^{*}}\right) \tau_{h} \\
& \left.-\frac{h_{P I} h_{I A^{*}}^{2}}{h_{A^{*} B^{*}}}\left(\tau_{h}\right)^{t} \varphi^{\prime \prime}\left(Q_{I A^{*}}\right) \tau_{h}\right)
\end{aligned}
$$


with $Q_{P I} \in[P I], \quad Q_{I A^{*}} \in\left[I A^{*}\right]$ and $Q_{I B^{*}} \in\left[I B^{*}\right]$.

Neglecting $T_{[P I], A^{*}}^{B^{*}}$ and exploiting Eq. (2.21) yields the following approximation:

$$
\begin{aligned}
-\int_{[P I]} \operatorname{grad} \varphi\left(K^{P} \xi_{[P I]}^{B^{*}}\right) d \gamma \approx & \frac{b_{h}\left(K^{P}\right) \widehat{a}_{h}\left(K^{L}\right) h_{P I}}{a_{h}\left(K^{P}\right) h_{I L}+\widehat{a}_{h}\left(K^{L}\right) h_{P I}}\left[\varphi_{P}-\varphi_{L}\right] \\
& +\frac{h_{P I}\left(d_{h}\left(K^{P}\right)\left\{a_{h}\left(K^{P}\right) h_{I L}+\widehat{a}_{h}\left(K^{L}\right) h_{P I}\right\}+b_{h}\left(K^{P}\right) h_{I L}\left\{\widehat{b}_{h}\left(K^{L}\right)-b_{h}\left(K^{P}\right)\right\}\right)}{h_{A^{*} B^{*}}\left[a_{h}\left(K^{P}\right) h_{I L}+\widehat{a}_{h}\left(K^{L}\right) h_{P I}\right]\left[\varphi_{B^{*}}-\varphi_{A^{*}}\right]^{-1}} .
\end{aligned}
$$

Similarly, let us focus on the computation of the flux across $[I L]$. For this purpose, we set

$K^{P} \xi_{[I L]}^{B^{*}}=-\widehat{c}_{h}\left(K^{L}\right) \sigma_{L}-\widehat{d}_{h}\left(K^{L}\right) \tau_{h}$

where the coefficients $\widehat{c}_{h}\left(K^{L}\right)$ and $\widehat{d}_{h}\left(K^{L}\right)$ are given by the following relations:

$$
\begin{aligned}
\widehat{c}_{h}\left(K^{L}\right) & =\widehat{b}_{h}\left(K^{L}\right), \quad \widehat{d}_{h}\left(K^{L}\right) \\
& =\frac{1}{\cos \theta_{h}^{L, I}}\left(\xi_{[I L]}^{B^{*}}\right)^{t} K^{L} \xi_{[I L]}^{B^{*}}>0 .
\end{aligned}
$$

So, the same ideas as those developed for the flux computation over $[P I]$ lead to

$$
\begin{aligned}
-\int_{[I L]} \operatorname{grad} \varphi\left(K^{L} \xi_{[I L]}^{B^{*}}\right) d \gamma \approx & \frac{\widehat{b}_{h}\left(K^{L}\right) a_{h}\left(K^{P}\right) h_{I L}}{a_{h}\left(K^{P}\right) h_{I L}+\widehat{a}_{h}\left(K^{L}\right) h_{P I}}\left[\varphi_{P}-\varphi_{L}\right] \\
& +\frac{h_{I L}\left(\widehat{d}_{h}\left(K^{L}\right)\left\{a_{h}\left(K^{P}\right) h_{I L}+\widehat{a}_{h}\left(K^{L}\right) h_{P I}\right\}+\widehat{b}_{h}\left(K^{L}\right) h_{P I}\left\{b_{h}\left(K^{P}\right)-\widehat{b}_{h}\left(K^{L}\right)\right\}\right)}{h_{A^{*} B^{*}}\left[a_{h}\left(K^{P}\right) h_{I L}+\widehat{a}_{h}\left(K^{L}\right) h_{P I}\right]\left[\varphi_{B^{*}}-\varphi_{A^{*}}\right]^{-1}}
\end{aligned}
$$

where truncation errors $T_{[I L], A^{*}}^{B^{*}}$ are neglected and read as

$$
\begin{aligned}
T_{[I L], A^{*}}^{B^{*}}= & \frac{\widehat{d}_{h}\left(K^{L}\right)}{2}\left(h_{I L}^{2}\left(\tau_{h}\right)^{t} \varphi^{\prime \prime}\left(Q_{I L}\right) \sigma_{L}\right. \\
& +\frac{h_{I L} h_{I B^{*}}^{2}}{h_{A^{*} B^{*}}}\left(\tau_{h}\right)^{t} \varphi^{\prime \prime}\left(Q_{I B^{*}}\right) \tau_{h} \\
& \left.-\frac{h_{I L} h_{I A^{*}}^{2}}{h_{A^{*} B^{*}}}\left(\tau_{h}\right)^{t} \varphi^{\prime \prime}\left(Q_{I A^{*}}\right) \tau_{h}\right)
\end{aligned}
$$

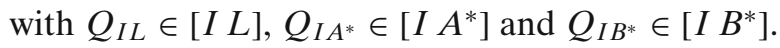

We have the following global estimate for the previous truncation errors:

Proposition 2.11 Under the same assumptions as those of Proposition 2.7, there exists a strictly positive number $C$, mesh independent, such that

$$
\left|T_{[P I], A^{*}}^{B^{*}}\right|+\left|T_{[I L], A^{*}}^{B^{*}}\right| \leq C h^{2} .
$$

\section{Step 3: Summary}

We summarize the flux approximation across the pseudo-edge $[P I L]$ in the following way:

$$
\begin{aligned}
Q_{[P I L]}^{B^{*}} \approx & \frac{b_{h}\left(K^{P}\right) \widehat{a}_{h}\left(K^{L}\right) h_{P I}+\widehat{b}_{h}\left(K^{L}\right) a_{h}\left(K^{P}\right) h_{I L}}{a_{h}\left(K^{P}\right) h_{I L}+\widehat{a}_{h}\left(K^{L}\right) h_{P I}} \\
& \times\left[\varphi_{P}-\varphi_{L}\right] \\
& +\frac{\omega_{h}(P, L, I)}{h_{A^{*} B^{*}}\left[a_{h}\left(K^{P}\right) h_{I L}+\widehat{a}_{h}\left(K^{L}\right) h_{P I}\right]} \\
& \times\left[\varphi_{B^{*}}-\varphi_{A^{*}}\right]
\end{aligned}
$$

where we have set

$$
\begin{aligned}
\omega_{h}(P, L, I)= & {\left[a_{h}\left(K^{P}\right) h_{I L}+\widehat{a}_{h}\left(K^{L}\right) h_{P I}\right] } \\
& \times\left[d_{h}\left(K^{P}\right) h_{P I}+\widehat{d}_{h}\left(K^{L}\right) h_{I L}\right] \\
& +h_{P I} h_{I L}\left[\widehat{b}_{h}\left(K^{L}\right)-b_{h}\left(K^{P}\right)\right] \\
& \times\left[b_{h}\left(K^{P}\right)-\widehat{b}_{h}\left(K^{L}\right)\right]
\end{aligned}
$$


Conclusion We deduce from the previous developments that the discrete balance equation in any dual cell $C_{B^{*}}$ reads

$$
\begin{aligned}
& \sum_{I \in \mathcal{E}^{B^{*}}} {\left[\frac{b_{h}\left(K^{P}\right) \widehat{a}_{h}\left(K^{L}\right) h_{P I}+\widehat{b}_{h}\left(K^{L}\right) a_{h}\left(K^{P}\right) h_{I L}}{a_{h}\left(K^{P}\right) h_{I L}+\widehat{a}_{h}\left(K^{L}\right) h_{P I}}\right.} \\
& \quad \times\left(\varphi_{P}-\varphi_{L}\right) \\
&\left.\quad+\frac{\omega_{h}(P, L, I)}{h_{A^{*} B^{*}}\left(a_{h}\left(K^{P}\right) h_{I L}+\widehat{a}_{h}\left(K^{L}\right) h_{P I}\right)}\left(\varphi_{B^{*}}-\varphi_{A^{*}}\right)\right] \\
& \quad \approx \int_{C_{B^{*}}} f(x) d x \quad \forall B^{*} \in \mathcal{D}
\end{aligned}
$$

where $\mathcal{E}^{B^{*}}$ is the set of edgepoints lying on the boundary of the dual cell $C_{B^{*}}$.

\subsubsection{Definition of the DDFV discrete problem}

From Eqs. (2.23) and (2.39), we define a DDFV formulation of Eqs. (1.1)-(1.2) as Find $\left\{\bar{\varphi}_{P}\right\}_{P \in \mathcal{P}}$ and $\left\{\bar{\varphi}_{D^{*}}\right\}_{D^{*} \in \mathcal{D}}$ such that

$$
\begin{aligned}
& \sum_{I \in \mathcal{E}^{P} \cap \mathcal{E}^{\text {int }}}\left[\frac{a_{h}\left(K^{P}\right) \widehat{a}_{h}\left(K^{L}\right) h_{A^{*} B^{*}}}{a_{h}\left(K^{P}\right) h_{I L}+\widehat{a}_{h}\left(K^{L}\right) h_{P I}}\left(\bar{\varphi}_{P}-\bar{\varphi}_{L}\right)\right. \\
& +\frac{\widehat{a}_{h}\left(K^{L}\right) b_{h}\left(K^{P}\right) h_{P I}+a_{h}\left(K^{P}\right) \widehat{b}_{h}\left(K^{L}\right) h_{I L}}{\widehat{a}_{h}\left(K^{L}\right) h_{P I}+a_{h}\left(K^{P}\right) h_{I L}} \\
& \left.\quad \times\left(\bar{\varphi}_{B^{*}}-\bar{\varphi}_{A^{*}}\right)\right] \\
& +\sum_{I \in \mathcal{E}^{P} \cap \mathcal{E}^{\mathrm{ext}}} a_{h}\left(K^{P}\right) \frac{h_{A^{*} B^{*}}}{h_{P I}} \bar{\varphi}_{P} \\
& =\int_{C_{P}} f(x) d x \quad \forall P \in \mathcal{P}
\end{aligned}
$$

$$
\begin{aligned}
& \sum_{J \in \mathcal{E}^{D^{*}}}\left[\frac{b_{h}\left(K^{G}\right) \widehat{a}_{h}\left(K^{H}\right) h_{G J}+\widehat{b}_{h}\left(K^{H}\right) a_{h}\left(K^{G}\right) h_{J H}}{a_{h}\left(K^{G}\right) h_{J H}+\widehat{a}_{h}\left(K^{H}\right) h_{G J}}\right. \\
& \quad \times\left(\bar{\varphi}_{G}-\bar{\varphi}_{H}\right) \\
& \left.\quad+\frac{\omega_{h}(G, H, J)}{h_{C^{*} D^{*}}\left(a_{h}\left(K^{G}\right) h_{J H}+\widehat{a}_{h}\left(K^{H}\right) h_{G J}\right)}\left(\bar{\varphi}_{D^{*}}-\bar{\varphi}_{C^{*}}\right)\right] \\
& \quad=\int_{C_{D^{*}}} f(x) d x \quad \forall D^{*} \in \mathcal{D}
\end{aligned}
$$

with: $\quad \bar{\varphi}_{E^{*}}=0 \quad \forall E^{*} \in \mathcal{D} \cap \partial \Omega$ where $(G, H) \in \mathcal{P} \times \mathcal{P}$ and $C^{*} \in \mathcal{D}$ are such that $\left[C^{*} D^{*}\right]$ defines the interface between the primary cells $C_{G}$ and $C_{H}$. Note that the definition of $\omega_{h}(G, H, J)$ is analogous to that of $\omega_{h}(P, L, I)$ (see relation (2.38)).

\subsubsection{Concluding remarks}

- Assuming that the primary mesh $\mathcal{P}$ is a square grid does not necessarily lead to a much simpler discrete system in the sense that the system matrix reduces to a 2 by 2 block diagonal matrix, even if $\mathcal{E}$ is made up of midedge points, and cellpoints are taken to be cell centers. This is exclusively due to the anisotropy of the porous medium $\Omega$. Indeed, if moreover the permeability matrix $K$ that governs the flow is taken to be a (strictly positive) real scalar (not necessarily uniformly constant in $\Omega$ ), then the system matrix reduces to a 2 by 2 block diagonal matrix. Moreover, each block corresponds to either cell-centered or vertex-centered finite volumes (see [30] for details).

- The DDFV technique exposed in this work applies to nonmatching grids (and especially to locally refined grids). Indeed, our approach is based upon the fact that any primary cell is a (convex) polygon and so is perfectly defined with a finite sequence of vertices clearly identified. Recall that a primary mesh is nonmatching if it possesses a cell, let us say $C$, with (at least) one side (i.e., one edge) denoted by $\left[A^{*} B^{*}\right]$ that involves a family of vertices $\left\{M_{z}^{*}\right\}_{1 \leq z \leq Z}$ such that $A^{*}$ and $B^{*}$ are different from $M_{z}^{*}$ for all $z \in\{1, \ldots, Z\}$. So, for any $z \in\{1, \ldots, Z\}, M_{z}^{*}$ is not associated with the primary cell $C$ but is associated with some cell $\widetilde{C} \in \mathcal{P}$ adjacent to $C$. Since $\left[A^{*} B^{*}\right]=\left[A^{*} M_{1}^{*}\right] \cup\left[M_{1}^{*} M_{2}^{*}\right] \cup$ $\left[M_{2}^{*} M_{3}^{*}\right] \cup \ldots \cup\left[M_{Z}^{*} B^{*}\right]$, the flux computation over the side $\left[A^{*} B^{*}\right]$ could be obtained as the sum of fluxes computed over the segments $\left[A^{*} M_{1}^{*}\right.$, $\left[M_{1}^{*} M_{2}^{*}\right],\left[M_{2}^{*} M_{3}^{*}\right], \ldots,\left[M_{Z}^{*} B^{*}\right]$. Since each of these segments involves an edgepoint from $\mathcal{E}$, let us say $I$, the corresponding pressure $\varphi_{I}$ may be eliminated from the expression of the approximate value of the exact flux $Q_{\left[M_{z}^{*} M_{z+1}^{*}\right]}^{C}$ over $\left[M_{z}^{*} M_{z+1}^{*}\right]$ (where $A^{*}=$ $M_{0}^{*}$ and $B^{*}=M_{Z+1}^{*}$ ), thanks to the flux continuity principle on the interface between the cells $C$ and $\widetilde{C}$.

Note that in the context of nonmatching grids, the construction of auxiliary and dual meshes meets the same rules as those imposed to a matching grid (in Section 2.1). Concerning the numerical validation of our DDFV approach for especially locally refined grids, we refer to Section 4 and more specifically to test problem no. 2 with rectangular 
nonconforming grids. The theoretical analysis on nonmatching grids of the DDFV method exposed in the current work is in preparation. Moreover, we should mention that a stability result and error estimates (for our DDFV method) have been proven in a forthcoming work on rectangular locally refined grids.

\subsection{Existence and uniqueness for a solution} of the discrete problem

Assume that all the cellpoints and all the interior vertices (with respect to the primary mesh) are numbered. On the other hand, $\operatorname{Card}(\mathcal{P})$ and $\operatorname{Card}(\mathcal{D})$ denote respectively the total number of cellpoints and interior vertices. The preliminary result is

Proposition 2.12 Under the assumptions (1.3)-(1.4), the matrix associated with the linear system (2.40)-(2.42) is symmetric and positive definite.

Proposition 2.13 Under the assumptions (1.3)-(1.4), the linear system (2.40)-(2.42) possesses a unique solution.

It is sufficient to prove Proposition 2.12 as it implies Proposition 2.13.

Proof It is easily seen that the symmetry of the matrix associated with the linear system (2.40)-(2.42) essentially follows from the symmetry of the diffusion coefficient $K$ (see assumption (1.3)). We should now prove the positive definiteness of that matrix. This will be done in three steps. Before starting, let us set

$\Phi_{c c}=\left\{\bar{\varphi}_{P}\right\}_{P \in \mathcal{P}} \quad$ and $\quad \Phi_{v c}=\left\{\bar{\varphi}_{D^{*}}\right\}_{D^{*} \in \mathcal{D}}$.

First step Development of the quadratic expression:

$\left[\Phi_{c c} \Phi_{v c}\right]\left[\begin{array}{ll}A & B \\ B^{t} & C\end{array}\right]\left[\begin{array}{l}\Phi_{c c} \\ \Phi_{v c}\end{array}\right]$

where $A$ is a $\operatorname{Card}(\mathcal{P}) \times \operatorname{Card}(\mathcal{P})$ symmetric matrix, $C$ is a $\operatorname{Card}(\mathcal{D}) \times \operatorname{Card}(\mathcal{D})$ symmetric matrix, $B$ is a $\operatorname{Card}(\mathcal{P}) \times \operatorname{Card}(\mathcal{D})$ matrix, and $B^{t}$ is the transpose form of $B$, which are the block elements of the system matrix. It is clear that

$$
\begin{aligned}
{\left[\Phi_{c c}\right.} & \left.\Phi_{v c}\right]\left[\begin{array}{cc}
A & B \\
B^{t} & C
\end{array}\right]\left[\begin{array}{c}
\Phi_{c c} \\
\Phi_{v c}
\end{array}\right] \\
= & \sum_{(G, H) \in \mathcal{P}^{2},\left(C^{*}, D^{*}\right) \in \mathcal{D}^{2} \text { with } \Gamma_{G} \cap \Gamma_{H}=\left[C^{*} D^{*}\right]} \\
& \times\left[\frac{h_{C^{*} D *} a_{h}\left(K^{G}\right) \widehat{a}_{h}\left(K^{H}\right)}{\widehat{a}_{h}\left(K^{H}\right) h_{G J}+a_{h}\left(K^{G}\right) h_{J H}}\left(\bar{\varphi}_{G}-\bar{\varphi}_{H}\right)^{2}\right. \\
& +\frac{\omega_{h}(G, H, J)}{h_{C^{*} D^{*}}\left\{\widehat{a}_{h}\left(K^{H}\right) h_{G J}+a_{h}\left(K^{G}\right) h_{J H}\right\}}\left(\bar{\varphi}_{C^{*}}-\bar{\varphi}_{D^{*}}\right)^{2} \\
& +2 \frac{\left\{\widehat{a}_{h}\left(K^{G}\right) b_{h}\left(K^{G}\right) h_{G J}+a_{h}\left(K^{G}\right) \widehat{b}_{h}\left(K^{H}\right) h_{J H}\right\}}{\widehat{a}_{h}\left(K^{H}\right) h_{G J}+a_{h}\left(K^{G}\right) h_{J H}} \\
& \left.\times\left(\bar{\varphi}_{G}-\bar{\varphi}_{H}\right)\left(\bar{\varphi}_{D^{*}}-\bar{\varphi}_{C^{*}}\right)\right] \\
& +\sum_{P \in \mathcal{P}}\left(\sum_{I \in \mathcal{E}^{P} \cap \mathcal{E}^{\mathrm{ext}}} a_{h}\left(K^{P}\right) \frac{h_{A^{*} B^{*}}}{h_{P I}}\right)\left(\bar{\varphi}_{P}\right)^{2}
\end{aligned}
$$

with the convention that

$\sum_{I \in \mathcal{E}^{P} \cap \mathcal{E}^{\mathrm{ext}}}=0 \quad$ if $\quad \mathcal{E}^{P} \cap \mathcal{E}^{\mathrm{ext}}=\emptyset$

where $\Gamma_{G}$ and $\Gamma_{H}$ represent respectively the boundaries of primary cells $C_{G}$ and $C_{H} ; J$ is the edge point lying on $\Gamma_{G} \cap \Gamma_{H}$, i.e., $J \in \mathcal{E}^{G} \cap \mathcal{E}^{H}$; and $A^{*}, B^{*} \in \mathcal{D}$ are such that $\Gamma_{P} \cap \Gamma=\left[A^{*} B^{*}\right]$.

Second step We should prove that the homogenized symmetric permeability matrix $K^{G H}$ is positive definite. For this purpose, define

$$
\begin{aligned}
K_{11}^{G H} & =\frac{h_{C^{*} D^{*}} a_{h}\left(K^{G}\right) \widehat{a}_{h}\left(K^{H}\right)}{\widehat{a}_{h}\left(K^{H}\right) h_{G J}+a_{h}\left(K^{G}\right) h_{J H}} \\
K_{22}^{G H} & =\frac{\omega_{h}(G, H, J)}{h_{C^{*} D^{*}}\left[\widehat{a}_{h}\left(K^{H}\right) h_{G J}+a_{h}\left(K^{G}\right) h_{J H}\right]} \\
K_{12}^{G H} & =K_{21}^{G H} \\
& =\frac{\widehat{a}_{h}\left(K^{H}\right) b_{h}\left(K^{G}\right) h_{G J}+a_{h}\left(K^{G}\right) \widehat{b}_{h}\left(K^{H}\right) h_{J H}}{\widehat{a}_{h}\left(K^{H}\right) h_{G J}+a_{h}\left(K^{G}\right) h_{J H}} .
\end{aligned}
$$


Then, we have

$$
\begin{aligned}
{\left[\Phi_{c c}\right.} & \left.\Phi_{v c}\right]\left[\begin{array}{cc}
A & B \\
B^{t} & C
\end{array}\right]\left[\begin{array}{l}
\Phi_{c c} \\
\Phi_{v c}
\end{array}\right]-\sum_{P \in \mathcal{P}} \\
\times & \left(\sum_{I \in \mathcal{E}^{P} \cap \mathcal{E}^{\mathrm{ext}}} a_{h}\left(K^{P}\right) \frac{h_{A^{*} B^{*}}}{h_{P I}}\right)\left(\bar{\varphi}_{P}\right)^{2} \\
= & \sum_{(G, H) \in \mathcal{P}^{2},\left(C^{*}, D^{*}\right) \in \mathcal{D}^{2} \text { with } \Gamma_{G} \cap \Gamma_{H}=\left[C^{*} D^{*}\right]} \\
& \times\left[K_{11}^{G H}\left(\bar{\varphi}_{G}-\bar{\varphi}_{H}\right)^{2}+K_{22}^{G H}\left(\bar{\varphi}_{D^{*}}-\bar{\varphi}_{C^{*}}\right)^{2}\right. \\
& \left.+2 K_{12}^{G H}\left(\bar{\varphi}_{G}-\bar{\varphi}_{H}\right)\left(\bar{\varphi}_{D^{*}}-\bar{\varphi}_{C^{*}}\right)\right] .
\end{aligned}
$$

Let us prove that the homogenized symmetric permeability tensor $K^{G H}$ is positive definite, i.e., $K_{11}^{G H} K_{22}^{G H}-$ $\left(K_{21}^{G H}\right)^{2}>0$. Setting

$\Delta^{G H}=K_{11}^{G H} K_{22}^{G H}-\left(K_{21}^{G H}\right)^{2}$,

it is easy to check that

$$
\begin{aligned}
\Delta^{G H}= & N_{1}\left[a_{h}\left(K^{G}\right) d_{h}\left(K^{G}\right)-\left(b_{h}\left(K^{G}\right)\right)^{2}\right] \\
& +N_{2}\left[\widehat{a}_{h}\left(K^{H}\right) \widehat{d}_{h}\left(K^{H}\right)-\left(\widehat{b}_{h}\left(K^{H}\right)\right)^{2}\right]
\end{aligned}
$$

where $N_{1}$ and $N_{2}$ are strictly positive numbers defined as

$$
\begin{aligned}
N_{1}= & \left(\frac{\widehat{a}_{h}\left(K^{H}\right) h_{G J}}{\widehat{a}_{h}\left(K^{H}\right) h_{G J}+a_{h}\left(K^{G}\right) h_{J H}}\right)^{2} \\
& +\frac{a_{h}\left(K^{G}\right) \widehat{a}_{h}\left(K^{H}\right) h_{G J} h_{J H}}{\left[\widehat{a}_{h}\left(K^{H}\right) h_{G J}+a_{h}\left(K^{G}\right) h_{J H}\right]^{2}}
\end{aligned}
$$

and

$$
\begin{aligned}
N_{2}= & \left(\frac{a_{h}\left(K^{G}\right) h_{J H}}{\widehat{a}_{h}\left(K^{H}\right) h_{G J}+a_{h}\left(K^{G}\right) h_{J H}}\right)^{2} \\
& +\frac{a_{h}\left(K^{G}\right) \widehat{a}_{h}\left(K^{H}\right) h_{G J} h_{J H}}{\left[\widehat{a}_{h}\left(K^{H}\right) h_{G J}+a_{h}\left(K^{G}\right) h_{J H}\right]^{2}} .
\end{aligned}
$$

Since the diffusion matrix $K$ is symmetric and positive definite (see assumptions (1.3)-(1.4)), the CauchySchwarz inequality for the inner product associated with $K$ ensures that

$a_{h}\left(K^{G}\right) d_{h}\left(K^{G}\right)-\left(b_{h}\left(K^{G}\right)\right)^{2}>0$

and

$\widehat{a}_{h}\left(K^{H}\right) \widehat{d}_{h}\left(K^{H}\right)-\left(\widehat{b}_{h}\left(K^{H}\right)\right)^{2}>0$ as either $\xi_{\left[C^{*} D^{*}\right]}^{G}$ and $\xi_{[G J]}^{D^{*}}$ or $\xi_{\left[C^{*} D^{*}\right]}^{G}$ and $\xi_{[J H]}^{D^{*}}$ are not collinear. Therefore, $\Delta^{G H}>0$ and thus $K^{G H}$ are symmetric and positive definite matrices.

Third step It follows from the previous step that the matrix $K^{G H}$ possesses strictly positive eigenvalues. Let $\lambda_{\min }^{G H}$ be its least eigenvalue. So, we have

$$
\begin{aligned}
& {\left[\Phi_{c c} \Phi_{v c}\right]\left[\begin{array}{ll}
A & B \\
B^{t} & C
\end{array}\right]\left[\begin{array}{l}
\Phi_{c c} \\
\Phi_{v c}
\end{array}\right]} \\
& \geq \sum_{P \in \mathcal{P}}\left(\sum_{I \in \mathcal{E}^{P} \cap \mathcal{E}^{\mathrm{ext}}} a_{h}\left(K^{P}\right) \frac{h_{A^{*} B^{*}}}{h_{P I}}\right)\left(\bar{\varphi}_{P}\right)^{2} \\
& \sum_{(G, H) \in \mathcal{P}^{2},\left(C^{*}, D^{*}\right) \in \mathcal{D}^{2} \text { with } \Gamma_{G} \cap \Gamma_{H}=\left[C^{*} D^{*}\right]} \\
& \times \lambda_{\min }^{G H}\left\{\left(\bar{\varphi}_{G}-\bar{\varphi}_{H}\right)^{2}+\left(\bar{\varphi}_{D^{*}}-\bar{\varphi}_{C^{*}}\right)^{2}\right\} \geq 0 .
\end{aligned}
$$

Thanks to the discrete boundary conditions in Eq. (2.42), the equality holds in Eq. (2.51) if and only if $\Phi_{c c}=0$ and $\Phi_{v c}=0$. Thus, the positive definiteness of the matrix $\left[\begin{array}{cc}A & B \\ B^{t} & C\end{array}\right]$ is proven.

\section{Stability and error estimates}

Fist of all, we assume that the primary mesh is regular in the sense of Definition 2.6. On the other hand, we use in what follows the terminology node as the generic name of cellpoints and vertices (with respect to the primary mesh).

\subsection{DDFV piecewise constant solutions}

Let us consider the auxiliary mesh $\mathcal{A}$ introduced in the previous section (see Fig. 2). Note that the closure of any cell of $\mathcal{A}$ contains one node and only one that may be inside $\Omega$ or on the boundary of $\Omega$.

Definition 3.1 An auxiliary mesh cell is degenerate if the corresponding node is lying on the boundary of $\Omega$.

As soon as the discrete unknowns (located at cellpoints and vertices with respect to the primary mesh) are computed, an important issue concerns the definition of an approximate solution to the continuous problem (1.1)-(1.2) in terms of function. 
- For addressing this issue, some authors have in the literature proposed the following: Let us denote by $\Upsilon_{T}$ the characteristic function of any cell $T$ (from primary, dual, or auxiliary mesh). Define almost everywhere in $\Omega$

$\bar{\varphi}^{\mathcal{P}}(x)=\sum_{P \in \mathcal{P}} \bar{\varphi}_{P} \Upsilon_{P}(x) \quad$ and

$\bar{\varphi}^{\mathcal{D}}(x)=\sum_{D \in \mathcal{D}} \bar{\varphi}_{D} \Upsilon_{D}(x)$.

Following those authors, the DDFV solution $\varphi^{\text {approx }}$ is defined as

$\varphi^{\operatorname{approx}}(x)=\frac{1}{2}\left[\bar{\varphi}^{\mathcal{P}}(x)+\bar{\varphi}^{\mathcal{D}}(x)\right]$

almost everywhere in $\Omega$.

Recall that $\varphi$ denotes the exact solution of the continuous problem (1.1)-(1.2) and set

$\varphi^{\mathcal{P}}(x)=\sum_{P \in \mathcal{P}} \varphi(P) \Upsilon_{P}(x) \quad$ and

$\varphi^{\mathcal{D}}(x)=\sum_{D \in \mathcal{D}} \varphi(D) \Upsilon_{D}(x)$.

Since one can show that (see Theorem 7.1 in [3], p. 27)

$\left\|\varphi^{\mathcal{P}}-\bar{\varphi}^{\mathcal{P}}\right\|_{L^{2}(\Omega)} \longrightarrow 0$ and

$\left\|\varphi^{\mathcal{D}}-\bar{\varphi}^{\mathcal{D}}\right\|_{L^{2}(\Omega)} \longrightarrow 0$ as $h \longrightarrow 0$,

it is obvious that $\left\|\frac{1}{2}\left[\varphi^{\mathcal{P}}+\varphi^{\mathcal{D}}\right]-\varphi^{\text {approx }}\right\|_{L^{2}(\Omega)} \longrightarrow 0$ as $h \longrightarrow 0$.

- We propose another formulation of the DDFV piecewise constant solution based upon the auxiliary mesh $\mathcal{A}$. The idea is to introduce the set $\mathcal{N}$ of nodes as the set made of cellpoints and vertices with respect to the primary mesh $\mathcal{P}$. Then, we define our DDFV piecewise constant solution $\bar{\varphi}_{h}$ almost everywhere in $\Omega$ as

$\bar{\varphi}_{h}(x)=\sum_{N \in \mathcal{N}} \bar{\varphi}_{N} \Upsilon_{N}(x)$.

It is our purpose in what follows to show that $\| \varphi^{\mathcal{A}}$ $\bar{\varphi}_{h} \|_{\mathcal{A}} \longrightarrow 0$ as $h \longrightarrow 0$. The $L^{2}$-convergence should follow from Lemma 3.2.

\subsection{A stability result}

We denote by $\mathbf{S}(\mathcal{A})$ the space of functions $v$ defined almost everywhere in $\Omega$ such that $v$ gets a constant value in any auxiliary cell, except in degenerate ones where it vanishes. Note that $\bar{\varphi}_{h} \in \mathbf{S}(\mathcal{A})$ and that the solution of the discrete system (2.40)-(2.42) could clearly be identified with $\bar{\varphi}_{h}$. We endow $\mathbf{S}(\mathcal{A})$ with the following discrete energy norm. For all $v \in \mathbf{S}(\mathcal{A})$, define

$$
\begin{aligned}
\|v\|_{\mathcal{A}}= & \sum_{P \in \mathcal{P} \text { such that } \Gamma_{P} \cap \mathcal{E}^{\mathrm{ext}} \neq \emptyset}\left(V_{P}\right)^{2} \\
& +\sum_{(G, H) \in \mathcal{P}^{2},\left(C^{*}, D^{*}\right) \in \mathcal{D}^{2}} \text { with } \Gamma_{G} \cap \Gamma_{H}=\left[C^{*} D^{*}\right] \\
& \left.\times\left[\left(V_{G}-V_{H}\right)^{2}+\left(V_{D^{*}}-V_{C^{*}}\right)^{2}\right]\right)^{\frac{1}{2}}
\end{aligned}
$$

where $V_{Y}$ is the constant value of $v \in \mathbf{S}(\mathcal{A})$ in the auxiliary cell associated with the node $Y$. We now analyze the stability (in the sense of the norm (3.4)) of the solution for Eqs. [2.40-2.42]. For this purpose, we need to introduce as in [30] a key result, namely a discrete version of Poincaré inequality based upon the following ingredients. Consider the linear operators defined by

$v \in \mathbf{S}(\mathcal{A}) \mapsto v^{\mathcal{P}} \in \mathbf{S}(\mathcal{P}) \quad$ and $\quad v \in \mathbf{S}(\mathcal{A}) \mapsto v^{\mathcal{D}} \in \mathbf{S}(\mathcal{D})$

where $\mathbf{S}(\mathcal{P})$ and $\mathbf{S}(\mathcal{D})$ are spaces of cellwise constant functions associated respectively with primary and dual grids and where

$v^{\mathcal{P}}(x)=\sum_{P \in \mathcal{P}} v_{P} \Upsilon_{P}(x) \quad$ and $\quad v^{\mathcal{D}}(x)=\sum_{D \in \mathcal{D}} v_{D} \Upsilon_{D}(x)$

a.e. in $\Omega$.

So $v^{\mathcal{P}}$ and $v^{\mathcal{D}}$ are in some sense projections of $v$ on $\mathbf{S}(\mathcal{P})$ and $\mathbf{S}(\mathcal{D})$, respectively. The function spaces $\mathbf{S}(\mathcal{P})$ and $\mathbf{S}(\mathcal{D})$ could respectively be equipped with the following discrete norms:

$$
\begin{aligned}
\left\|v^{\mathcal{P}}\right\|_{\mathcal{P}}= & \sum_{P \in \mathcal{P} \text { such that } \Gamma_{P} \cap \mathcal{E}^{\text {ext } \neq \emptyset}}\left(V_{P}\right)^{2} \\
& \left.+\sum_{(G, H) \in \mathcal{P}^{2} \text { with } \Gamma_{G} \cap \Gamma_{H} \in \mathcal{I}_{\mathcal{P}}}\left(V_{G}-V_{H}\right)^{2}\right)^{\frac{1}{2}}
\end{aligned}
$$

and

$$
\left\|v^{\mathcal{D}}\right\|_{\mathcal{D}}=\left(\sum_{\left(C^{*}, D^{*}\right) \in \mathcal{D}^{2}} \text { with }\left[C^{*} D^{*}\right] \in \mathcal{I}_{\mathcal{P}}\left(V_{C^{*}}-V_{D^{*}}\right)^{2}\right)^{\frac{1}{2}}
$$


where $\mathcal{I}_{\mathcal{P}}$ represents the set of primary mesh interfaces. The following lemmas are the key ingredients for our purpose:

Lemma 3.2 (Discrete version of Poincaré inequality) There exists a strictly positive number $C$, not depending on $h$, such that

$$
\left\|v^{\mathcal{P}}\right\|_{L^{2}(\Omega)}+\left\|v^{\mathcal{D}}\right\|_{L^{2}(\Omega)} \leq C\|v\|_{\mathcal{A}} \quad \forall v \in \mathbf{S}(\mathcal{A}) .
$$

Remark 3.3 In the framework of DDFV analysis of 2D linear and nonlinear elliptic problems, the inequality (3.8) is well known. In a general case where $L^{2}(\Omega)$ is replaced by $L^{P}(\Omega)$, with $1<P<+\infty$, the proof can, for instance, be found in [3] (see also [4] or Proposition 4.5 in [9] at p. 15).

Lemma 3.4 Consider the symmetric positive definite matrix $K^{G H}$ (where $G, H \in \mathcal{P}$ are such that $\Gamma_{G} \cap \Gamma_{H} \in$ $\mathcal{E})$ defined by the relations in Eq. (2.44). Under the conditions (2.12) and (2.13), there exists a strictly positive real number $\mu$, mesh independent, such that the least eigenvalue $\lambda_{\min }^{G H}$ of the matrix $K^{G H}$ satisfies to $\mu \preceq$ $\lambda_{\min }^{G H}$.

Proof The eigenvalues $\lambda$ of the symmetric positive definite matrix $K^{G H}$ satisfy to the so-called characteristic equation associated with $K^{G H}$, i.e.,

$\lambda^{2}-\left[K_{11}^{G H}+K_{22}^{G H}\right] \lambda+\left[K_{11}^{G H} K_{22}^{G H}-\left(K_{12}^{G H}\right)^{2}\right]=0$.

The least eigenvalue of $K^{G H}$ denoted by $\lambda_{\min }^{G H}$ is given by the relation

$\lambda_{\min }^{G H}=\frac{\left[K_{11}^{G H}+K_{22}^{G H}\right]-\sqrt{\Delta}}{2}$

where $\Delta=\left[K_{11}^{G H}+K_{22}^{G H}\right]^{2}-4\left[K_{11}^{G H} K_{22}^{G H}-\left(K_{12}^{G H}\right)^{2}\right]$ is a strictly positive number. One can easily deduce that

$\lambda_{\min }^{G H} \succeq \frac{\operatorname{det}\left(K^{G H}\right)}{\left[K_{11}^{G H}+K_{22}^{G H}\right]+\operatorname{det}\left(K^{G H}\right)+1}$

where $\operatorname{det}\left(K^{G H}\right)=K_{11}^{G H} K_{22}^{G H}-\left(K_{12}^{G H}\right)^{2}$ is a strictly positive number. We should bound the quantities $K_{11}^{G H}, K_{22}^{G H}$ and $\operatorname{det}\left(K^{G H}\right)$ by strictly positive, mesh independent, real numbers. Let us start first with $\operatorname{det}\left(K^{G H}\right)$. We consider a change of coordinates by moving from the initial Cartesian coordinates to a local one, namely $\left(J, \frac{\overrightarrow{C^{*} D^{*}}}{\left|\overrightarrow{C^{*} D^{*}}\right|}, \xi_{\left[C^{*} D^{*}\right]}^{\perp}\right)$, where $J$ is the edgepoint fixed on the interface $\left[C^{*} D^{*}\right]$ between the cells $C_{G}$ and $C_{H}$ and where $\xi_{\left[C^{*} D^{*}\right]}^{\perp}$ is a vector orthogonal to
$\overrightarrow{C^{*} D^{*}}$ and oriented such that the basis change matrix $M$ is a rotation. Denoting the permeability tensor of the cell $C_{G}$ by $K^{G}=\left\{K^{G} i\right\}$ in the initial Cartesian coordinates, and by $\widetilde{K}^{G}=\left\{\widetilde{K}_{i j}^{G}\right\}$ in the local coordinates, we have $\widetilde{K}^{G}=M^{-1} K^{G} M$. Similarly, we get $\widetilde{K}^{H}=$ $M^{-1} K^{H} M$ for the cell $C_{H}$. Then, it is easy to check that

$a_{h}\left(K^{G}\right) d_{h}\left(K^{G}\right)-\left[b_{h}\left(K^{G}\right)\right]^{2}=\widetilde{K}_{11}^{G} \widetilde{K}_{22}^{G}-\left[\tilde{K}_{12}^{G}\right]^{2}$,

i.e.,

$a_{h}\left(K^{G}\right) d_{h}\left(K^{G}\right)-\left[b_{h}\left(K^{G}\right)\right]^{2}=\operatorname{det}\left(\tilde{K}^{G}\right)=\operatorname{det}\left(K^{G}\right)$

where $\operatorname{det}($.$) denotes the determinant. Similarly, we$ have for the cell $C_{H}$

$\widehat{a}_{h}\left(K^{H}\right) \widehat{d}_{h}\left(K^{H}\right)-\left[\widehat{b}_{h}\left(K^{H}\right)\right]^{2}=\operatorname{det}\left(\widetilde{K}^{H}\right)=\operatorname{det}\left(K^{H}\right)$.

It follows from what precedes that

$\operatorname{det}\left(K^{G H}\right)=N_{1} \operatorname{det}\left(K^{G}\right)+N_{2} \operatorname{det}\left(K^{H}\right)$

where $N_{1}$ and $N_{2}$ are given by the relations (2.47) and (2.48).

On one hand, we can deduce from assumptions (1.4), (2.12), and (2.13) that

$N_{i} \succeq \frac{\varpi^{2}}{2}\left[\frac{\gamma_{\min }}{\gamma_{\max }}\right]^{2} \sin \theta \quad \forall i=1,2$.

On the other hand, we remark that

$\operatorname{det}\left(K^{P}\right) \succeq \min \left\{\operatorname{det}\left(K^{s}\right), s \in S\right\} \quad \forall P \in \mathcal{P}$

where the set $S$ (introduced in Section 2.1, Eq. [2.1]) depends exclusively on the lithologic structure of the medium $\Omega$. Then, we deduce that

$\operatorname{det}\left(K^{G H}\right) \succeq \varpi^{2}\left[\frac{\gamma_{\min }}{\gamma_{\max }}\right]^{2} \sin \theta\left[\min \left\{\operatorname{det}\left(K^{s}\right), s \in S\right\}\right]$.

Remarking that

$\operatorname{det}\left(K^{G H}\right) \preceq K_{11}^{G H} K_{22}^{G H}+\left(K_{12}^{G H}\right)^{2}$

and exploiting again the assumptions (1.4), (2.12), and (2.13) leads to the following inequality:

$\operatorname{det}\left(K^{G H}\right) \preceq \frac{2\left(\gamma_{\max }\right)^{4} \max \{2, \varpi\}}{\varpi^{3}\left(\gamma_{\min }\right)^{2}(\sin \theta)^{4}}$. 
Thanks again to Eqs. (1.4), (2.12), and (2.13), one can easily check that

$K_{11}^{G H}+K_{22}^{G H} \preceq\left(\frac{\gamma_{\max }}{\sqrt{2} \varpi \sin \theta}\right)^{2}\left[1+\frac{2}{\varpi}+\frac{4 \gamma_{\max }}{\varpi \sin \theta}\right]$.

Lemma 3.4 follows from the combination of Eqs. (3.17), (3.18), and (3.19).

Let us give now one of the main results of this section.

Proposition 3.5 (A stability result) Assume that the condition (2.13) is fulfilled and that the mesh system $(\mathcal{P}, \mathcal{E})$ is regular in the sense of Definition 2.6, i.e., the condition (2.12) is satisfied. Then, the piecewise constant solution $\bar{\varphi}_{h}$ of the problem (1.1)-(1.2) obeys the inequality

$\left\|\bar{\varphi}_{h}\right\|_{\mathcal{A}} \leq C\|f\|_{L^{2}(\Omega)}$

where $C$ is a strictly positive, mesh-independent real number.

Proof Multiplying Eq. (2.40) by $\bar{\varphi}_{P}$ and Eq. (2.41) by $\bar{\varphi}_{D^{*}}$ and summing over $P \in \mathcal{P}$ and $D^{*} \in \mathcal{D}$ respectively leads to

$\left[\begin{array}{ll}\Phi_{c c} & \Phi_{v c}\end{array}\right]\left[\begin{array}{cc}A & B \\ B^{t} & C\end{array}\right]\left[\begin{array}{l}\Phi_{c c} \\ \Phi_{v c}\end{array}\right]=\left[\begin{array}{ll}U_{c c} & U_{v c}\end{array}\right]\left[\begin{array}{l}F_{c c} \\ F_{v c}\end{array}\right]$

Set: $\quad L H S=\left[\Phi_{c c} \Phi_{v c}\right]\left[\begin{array}{ll}A & B \\ B^{t} & C\end{array}\right]\left[\begin{array}{l}\Phi_{c c} \\ \Phi_{v c}\end{array}\right]$

$$
R H S=\left[\Phi_{c c} \Phi_{v c}\right]\left[\begin{array}{l}
\Phi_{c c} \\
\Phi_{v c}
\end{array}\right]
$$

We know from Lemma 3.4 that under the conditions (2.12) and (2.13), there exists a strictly positive, meshindependent number $\alpha$ such that

$\alpha\left\|\bar{\varphi}_{h}\right\|_{\mathcal{A}}^{2} \leq L H S$.

On the other hand, we have

$\frac{1}{2}\{R H S\}^{2} \leq\left|\sum_{P \in \mathcal{P}} \int_{C_{P}} f \bar{\varphi}_{P}\right|^{2}+\left|\sum_{D^{*} \in \mathcal{D}} \int_{C_{D^{*}}} f \bar{\varphi}_{D^{*}}\right|^{2}$.
We get from the Cauchy-Schawrz inequality that

$$
\begin{gathered}
\left|\sum_{P \in \mathcal{P}} \int_{C_{P}} f \bar{\varphi}_{P}\right|^{2} \leq\|f\|_{L^{2}(\Omega)}^{2}\left\|\bar{\varphi}_{h}^{\mathcal{P}}\right\|_{L^{2}(\Omega)}^{2} \text { and } \\
\left|\sum_{D^{*} \in \mathcal{D}} \int_{C_{D^{*}}} f \bar{\varphi}_{D^{*}}\right|^{2} \leq\|f\|_{L^{2}(\Omega)}^{2}\left\|\bar{\varphi}_{h}^{\mathcal{D}}\right\|_{L^{2}(\Omega)}^{2} .
\end{gathered}
$$

Therefore,

$R H S \leq \sqrt{2}\|f\|_{L^{2}(\Omega)}\left\{\left\|\bar{\varphi}_{h}^{\mathcal{P}}\right\|_{L^{2}(\Omega)}^{2}+\left\|\bar{\varphi}_{h}^{\mathcal{D}}\right\|_{L^{2}(\Omega)}^{2}\right\}^{\frac{1}{2}}$.

Comparing Eq. (3.22) with Eq. (3.23) and considering Lemma3.2 leads to the end of the proof of Proposition 3.5.

\subsection{Error estimates for the DDFV solution $\bar{\varphi}_{h}$}

Following the ideas developed in [30], we investigate here error estimates (in the sense of the discrete energy norm (3.4)) for the piecewise constant approximate solution $\bar{\varphi}_{h}$.

\subsubsection{Exact nodal potentials as a solution of a discrete system}

Adding the truncation error to the flux approximation (2.22) across $\left[A^{*} B^{*}\right]=\Gamma_{P} \cap \Gamma_{L}$ leads to the following identity:

$$
\begin{aligned}
Q_{\left[A^{*} B^{*}\right]}^{P}= & {\left[\frac{a_{h}\left(K^{P}\right) \widehat{a}_{h}\left(K^{L}\right) h_{A^{*} B^{*}}}{a_{h}\left(K^{P}\right) h_{I L}+\widehat{a}_{h}\left(K^{L}\right) h_{P I}}\right]\left[\varphi_{P}-\varphi_{L}\right] } \\
& +\left[\frac{\widehat{a}_{h}\left(K^{L}\right) b_{h}\left(K^{P}\right) h_{P I}+a_{h}\left(K^{P}\right) \widehat{b}_{h}\left(K^{L}\right) h_{I L}}{\widehat{a}_{h}\left(K^{L}\right) h_{P I}+a_{h}\left(K^{P}\right) h_{I L}}\right] \\
& \times\left[\varphi_{B^{*}}-\varphi_{A^{*}}\right]+R_{I\left(A^{*}, B^{*}, L\right)}^{P}
\end{aligned}
$$

where $I$ is the edgepoint fixed in $\left[A^{*} B^{*}\right]$ and where the truncation error $R_{I\left(A^{*}, B^{*}, L\right)}^{P}$ is defined by

$$
\begin{aligned}
R_{I\left(A^{*}, B^{*}, L\right)}^{P}= & {\left[1+\frac{a_{h}\left(K^{P}\right) h_{I L}}{a_{h}\left(K^{P}\right) h_{I L}+\widehat{a}_{h}\left(K^{L}\right) h_{P I}}\right] T_{I\left(A^{*}, B^{*}\right)}^{P} } \\
& +\frac{a_{h}\left(K^{P}\right) h_{I L}}{a_{h}\left(K^{P}\right) h_{I L}+\widehat{a}_{h}\left(K^{L}\right) h_{P I}} T_{I\left(A^{*}, B^{*}\right)}^{L} .
\end{aligned}
$$


Similarly, according to Eq. (2.37), the exact flux across [PIL] (part of the boundary of the dual cell $C_{B^{*}}$ ) meets the identity

$$
\begin{aligned}
Q_{[P I L]}^{B^{*}}= & \frac{b_{h}\left(K^{P}\right) \widehat{a}_{h}\left(K^{L}\right) h_{P I}+\widehat{b}_{h}\left(K^{L}\right) a_{h}\left(K^{P}\right) h_{I L}}{a_{h}\left(K^{P}\right) h_{I L}+\widehat{a}_{h}\left(K^{L}\right) h_{P I}} \\
& \times\left[\varphi_{P}-\varphi_{L}\right] \\
& +\frac{\omega_{h}(P, L, I)}{h_{A^{*} B^{*}}\left[a_{h}\left(K^{P}\right) h_{I L}+\widehat{a}_{h}\left(K^{L}\right) h_{P I}\right]} \\
& \times\left[\varphi_{B^{*}}-\varphi_{A^{*}}\right]+R_{I\left(P, L, A^{*}\right)}^{B^{*}}
\end{aligned}
$$

where the truncation error $R_{I\left(P, L, A^{*}\right)}^{B^{*}}$ is given by

$$
\begin{aligned}
& R_{I\left(P, L, A^{*}\right)}^{B^{*}} \\
& =T_{[P I], A^{*}}^{B^{*}}+T_{[I L], A^{*}}^{B^{*}} \\
& \quad+\frac{\left\{T_{I\left(A^{*}, B^{*}\right)}^{P}+T_{I\left(A^{*}, B^{*}\right)}^{L}\right\}\left\{\widehat{b}_{h}\left(K^{L}\right)-b_{h}\left(K^{P}\right)\right\}}{a_{h}\left(K^{P}\right) \frac{h_{A^{*} B^{*}}}{h_{P I}}+\widehat{a}_{h}\left(K^{L}\right) \frac{h_{A^{*} B^{*}}}{h_{I L}}} .
\end{aligned}
$$

Let us give some estimates related to flux errors not only for the set of primal edges but also for the set of dual edges.

Proposition 3.6 $\left(L^{\infty}\right.$-Estimates for flux errors over primary and dual edges) Under the same assumptions as those of Proposition 2.7, the truncation errors $R_{I\left(A^{*}, B^{*}, L\right)}^{P}$ and $R_{I\left(P, L, A^{*}\right)}^{B^{*}}$ associated respectively with flux approximations across any primary edge $\left[A^{*} B^{*}\right]$ and over the corresponding dual edge [PIL] meet the following inequalities:

$$
\left|R_{I\left(A^{*}, B^{*}, L\right)}^{P}\right| \leq C h^{2} \quad \text { and } \quad\left|R_{I\left(P, L, A^{*}\right)}^{B^{*}}\right| \leq C h^{2} .
$$

Note that we mean by corresponding dual edge the unique pseudo-edge that intersects with the primary edge $\left[A^{*} B^{*}\right]$.

Proof It essentially follows from Proposition 2.7 that

$$
\left|R_{I\left(A^{*}, B^{*}, L\right)}^{P}\right| \leq C h^{2}
$$

On the other hand, according to Proposition 2.7 and Proposition 2.11, the following inequality holds:

$\left|R_{I\left(P, L, A^{*}\right)}^{B^{*}}\right| \leq C h^{2}$.

Remark 3.7 The inequalities in Eq. (3.28) may be written in the following compact forms: Start with defining the flux error vectors $R^{\mathcal{P}}$ and $R^{\mathcal{D}}$ by $R^{\mathcal{P}}=\left(R_{e}^{\mathcal{P}}\right)_{e \in \mathcal{E}}$, with $R_{e}^{\mathcal{P}}$ of the form $R_{I\left(A^{*}, B^{*}, L\right)}^{P}$, and by $R^{\mathcal{D}}=\left(R_{e}^{\mathcal{D}}\right)_{e \in \mathcal{E}^{*}}$, with $R_{e}^{\mathcal{D}}$ of the form $R_{I\left(P, L, A^{*}\right)}^{B^{*}}$, where $\mathcal{E}^{*}$ denotes the set of pseudo-edges. Then, the following estimates hold:

$\max _{e \in \mathcal{E}}\left|R_{e}^{\mathcal{P}}\right| \leq C h^{2} \quad$ and $\quad \max _{e \in \mathcal{E}^{*}}\left|R_{e}^{\mathcal{D}}\right| \leq C h^{2}$.

All the ingredients are gathered for writing the system of equations satisfied by the vector of exact nodal potentials $\left\{\varphi_{P}\right\}_{P \in \mathcal{P}}$ and $\left\{\varphi_{D^{*}}\right\}_{D^{*} \in \mathcal{D}}$ :

$$
\begin{aligned}
& \sum_{I \in \mathcal{E}^{P} \cap \mathcal{E}^{\text {int }}}\left[\frac{a_{h}\left(K^{P}\right) \widehat{a}_{h}\left(K^{L}\right) h_{A^{*} B^{*}}}{a_{h}\left(K^{P}\right) h_{I L}+\widehat{a}_{h}\left(K^{L}\right) h_{P I}}\left(\varphi_{P}-\varphi_{L}\right)\right. \\
& \left.+\frac{\widehat{a}_{h}\left(K^{L}\right) b_{h}\left(K^{P}\right) h_{P I}+a_{h}\left(K^{P}\right) \widehat{b}_{h}\left(K^{L}\right) h_{I L}}{\widehat{a}_{h}\left(K^{L}\right) h_{P I}+a_{h}\left(K^{P}\right) h_{I L}}\left(\varphi_{B^{*}}-\varphi_{\left.A^{*}\right)}\right)\right] \\
& +\sum_{I \in \mathcal{E}^{P} \cap \mathcal{E}^{\mathrm{ext}}} a_{h}\left(K^{P}\right) \frac{h_{A^{*} B^{*}}}{h_{P I}} \varphi_{P} \\
& =\int_{C_{P}} f(x) d x-\sum_{I \in \mathcal{E}^{P} \cap \mathcal{E}^{\text {int }}} R_{I\left(A^{*}, B^{*}, L\right)} \\
& \quad-\sum_{I \in \mathcal{E}^{P} \cap \mathcal{E}^{\mathrm{ext}}} T_{I\left(A^{*}, B^{*}\right)}^{P} \quad \forall P \in \mathcal{P}
\end{aligned}
$$

$$
\begin{aligned}
& \sum_{J \in \mathcal{E}^{D^{*}}}\left[\frac{b_{h}\left(K^{G}\right) \widehat{a}_{h}\left(K^{H}\right) h_{G J}+\widehat{b}_{h}\left(K^{H}\right) a_{h}\left(K^{G}\right) h_{J H}}{a_{h}\left(K^{G}\right) h_{J H}+\widehat{a}_{h}\left(K^{H}\right) h_{G J}}\right. \\
& \quad \times\left(\varphi_{G}-\varphi_{H}\right) \\
& \left.+\frac{\omega_{h}(G, H, J)}{h_{C^{*} D^{*}}\left(a_{h}\left(K^{G}\right) h_{J H}+\widehat{a}_{h}\left(K^{H}\right) h_{G J}\right)}\left(\varphi_{D^{*}}-\varphi_{C^{*}}\right)\right] \\
& =\int_{C_{D^{*}}} f(x) d x-\sum_{J \in \mathcal{E}^{D^{*}}} R_{J\left(G, H, C^{*}\right)}^{D^{*}} \quad \forall D^{*} \in \mathcal{D}
\end{aligned}
$$

with

$\varphi_{E^{*}}=0 \quad \forall E^{*} \in \mathcal{D} \cap \partial \Omega$

where $(G, H) \in \mathcal{P} \times \mathcal{P}$ and $C^{*} \in \mathcal{D}$ are such that $\left[C^{*} D^{*}\right]$ defines the interface between the primary cells $C_{G}$ and $C_{H}$. See Eq. (2.38) for the definition of $\omega_{h}(P, L, I)$ and define similarly $\omega_{h}(G, H, J)$.

\subsubsection{Error analysis of the piecewise constant solution $\bar{\varphi}_{h}$}

Our purpose in this subsection is to investigate some error estimates (for convenient norms) when $\bar{\varphi}_{h}$ is taken to be an approximate solution of the diffusion problem (1.1)-(1.2). Due to the conservativity of the 
flux approximations, the truncation errors obey to the following relation:

$R_{I(.,,, b)}^{a}+R_{I(.,,, a)}^{b}=0 \quad \forall I \in \mathcal{E}^{\mathrm{int}}$,

with $\mathcal{E}^{a} \cap \mathcal{E}^{b}=\{I\}$

where $a$ and $b$ are two cellpoints or vertices and $\mathcal{E}^{a}$ and $\mathcal{E}^{b}$ are sets of edgepoints respectively associated with $a$ and $b$ (that also represent two primary or dual adjacent cells: see Remark 2.5).

The notion of diamond cell plays a key role in what follows. So, let us now introduce this notion after what follows. We mean by adjacent cellpoints two cellpoints lying in two adjacent primary cells.

Definition 3.8 Given two adjacent cellpoints $P$ and $L$ sharing $\left[A^{*} B^{*}\right]$ as common interface, the quadrangle $\left(P, A^{*}, L, B^{*}\right)$ defines a diamond cell. The set of diamond cells is a diamond mesh denoted by $\mathcal{M}$ in the sequel.

Note that each diamond cell is actually associated with one and only one interface $\left[A^{*} B^{*}\right]$. When $\left[A^{*} B^{*}\right]$ is lying on the domain boundary, the corresponding diamond cell is degenerate since it is defined as a triangle (see illustrations in Fig. 6). The following assumption plays a key role in what follows:

$\exists v, \zeta \in \mathbb{R}_{+}^{*} \quad$ such that: $v h^{2} \leq \operatorname{meas}(M) \leq \zeta h^{2}$

$$
\forall M \in \mathcal{M}
$$

where $v$ and $\zeta$ are strictly positive, mesh-independent numbers and meas(.) is the Lebesgue measure in any spatial dimension. Let us set

$E_{P}=\varphi_{P}-\bar{\varphi}_{P}$, for $P \in \mathcal{P} \quad$ and $\quad E_{D^{*}}=\varphi_{D^{*}}-\bar{\varphi}_{D^{*}}$, for $D^{*} \in \mathcal{D}$.
An adequate linear combination of Eqs. (3.36)-(3.38) with Eqs. (2.40)-(2.42) yields

$$
\begin{aligned}
& \sum_{I \in \mathcal{E}^{P} \cap \mathcal{E}^{\text {int }}}\left(\frac{a_{h}\left(K^{P}\right) \widehat{a}_{h}\left(K^{L}\right) h_{A^{*} B^{*}}}{a_{h}\left(K^{P}\right) h_{I L}+\widehat{a}_{h}\left(K^{L}\right) h_{P I}}\left(E_{P}-E_{L}\right)\right. \\
& +\frac{\widehat{a}_{h}\left(K^{L}\right) b_{h}\left(K^{P}\right) h_{P I}+a_{h}\left(K^{P}\right) \widehat{b}_{h}\left(K^{L}\right) h_{I L}}{\widehat{a}_{h}\left(K^{L}\right) h_{P I}+a_{h}\left(K^{P}\right) h_{I L}} \\
& \quad \times\left(E_{B^{*}}-E_{\left.\left.A^{*}\right)\right)}\right. \\
& +\sum_{I \in \mathcal{E}^{P} \cap \mathcal{E}^{\mathrm{ext}}} a_{h}\left(K^{P}\right) \frac{h_{A^{*} B^{*}}}{h_{P I}} E_{P} \\
& =-\sum_{I \in \mathcal{E}^{P} \cap \mathcal{E}^{\text {int }}} R_{I\left(A^{*}, B^{*}, L\right)}^{P} \quad \forall P \in \mathcal{P} \\
& -\sum_{I \in \mathcal{E}^{P} \cap \mathcal{E}^{\mathrm{ext}}} T_{I\left(A^{*}, B^{*}\right)}^{P} \quad \forall P
\end{aligned}
$$

$$
\begin{aligned}
& \sum_{J \in \mathcal{E}^{D^{*}}}\left[\frac{b_{h}\left(K^{G}\right) \widehat{a}_{h}\left(K^{H}\right) h_{G J}+\widehat{b}_{h}\left(K^{H}\right) a_{h}\left(K^{G}\right) h_{J H}}{a_{h}\left(K^{G}\right) h_{J H}+\widehat{a}_{h}\left(K^{H}\right) h_{G J}}\right. \\
& \quad \times\left(E_{G}-E_{H}\right) \\
& \left.\quad+\frac{\omega_{h}(G, H, J)}{h_{C^{*} D^{*}}\left(a_{h}\left(K^{G}\right) h_{J H}+\widehat{a}_{h}\left(K^{H}\right) h_{G J}\right)}\left(E_{D^{*}}-E_{C^{*}}\right)\right] \\
& =-\sum_{J \in \mathcal{E}^{D^{*}}} R_{J\left(G, H, C^{*}\right)}^{D^{*}} \quad \forall D^{*} \in \mathcal{D}
\end{aligned}
$$

with

$E_{Q^{*}}=0 \quad \forall Q^{*} \in \mathcal{D} \cap \partial \Omega$.

Remark that the quantities $\left\{E_{P}\right\}_{P \in \mathcal{P}}$ and $\left\{E_{D^{*}}\right\}_{D^{*} \in \mathcal{D}}$ can be viewed as values in auxiliary cells of a function $E_{h} \in \mathbf{S}(\mathcal{A})$. Note that these quantities are solution to a linear system of the same type as Eqs. (2.40)-(2.42) in the sense that both of them are associated with the

Fig. 6 Left: A normal diamond cell. Right: A degenerate diamond cell
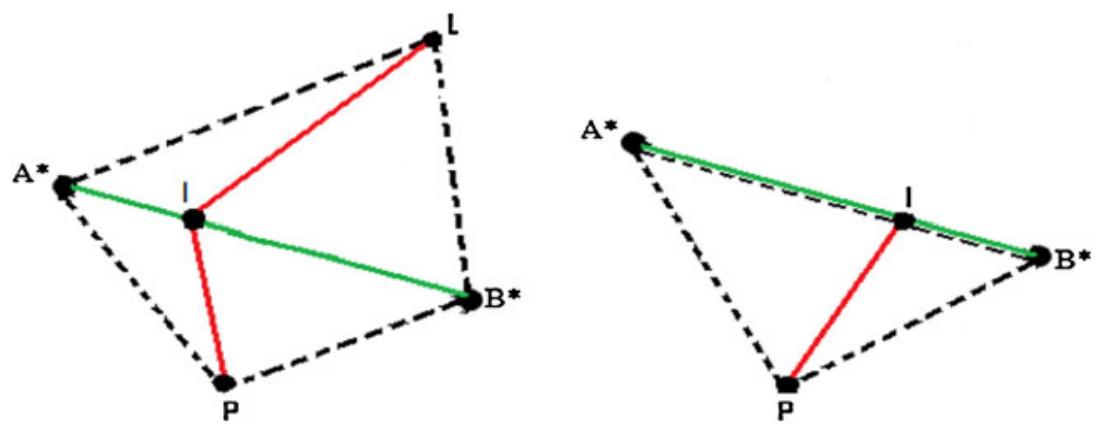
same matrix. Consequently, it follows from Lemma 3.4 and scheme conservativity that

$$
\begin{aligned}
\delta\left\|E_{h}\right\|_{\mathcal{A}}^{2} \leq & \sum_{I\left(P, L, A^{*}, B^{*}\right) \in \mathcal{E}^{\text {int }}} R_{I\left(P, L, A^{*}, B^{*}\right)}^{\max } \\
& \times\left(\left|E_{P}-E_{L}\right|+\left|E_{B^{*}}-E_{A^{*}}\right|\right) \\
& +\sum_{I\left(P, A^{*}, B^{*}\right) \in \mathcal{E}^{\mathrm{ext}}}\left|T_{I\left(P, A^{*}, B^{*}\right)}^{P}\right|\left|E_{P}\right|
\end{aligned}
$$

where we have set

$R_{I\left(P, L, A^{*}, B^{*}\right)}^{\max }=\max \left\{\left|R_{I\left(A^{*}, B^{*}, L\right)}^{P}\right|,\left|R_{I\left(P, L, A^{*}\right)}^{B^{*}}\right|\right\}$

and where $\delta$ is some strictly positive number that is mesh independent. Due to Eqs. (3.30) and (3.29), there exists $\widehat{C}>0$, mesh independent, such that

$0 \leq R_{I\left(P, L, A^{*}, B^{*}\right)}^{\max } \leq \widehat{C} h^{2}$.

On the other hand, define the following:

$\mathcal{S}_{I\left(P, L, A^{*}, B^{*}\right)}=2 \mathrm{D}$ Lebesgue measure of the diamond cell defined by the points $P, L, A^{*}$, and $B^{*}$ and associated with $I \in \mathcal{E}^{\text {int }}$,

$\mathcal{S}_{I\left(P, A^{*}, B^{*}\right)}=2 \mathrm{D}$ Lebesgue measure of the degenerate diamond cell defined by the points $P, A^{*}$, and $B^{*}$ and associated with $I \in \mathcal{E}^{\text {ext }}$.

Therefore, thanks to the Cauchy-Schawrz inequality, it follows from Eq. (3.39) that

$$
\begin{aligned}
\delta\left\|E_{h}\right\|_{\mathcal{A}}^{2} \leq & \left(\sum_{I\left(P, L, A^{*}, B^{*}\right) \in \mathcal{E}^{\mathrm{int}}} \mathcal{S}_{I\left(P, L, A^{*}, B^{*}\right)}\right. \\
& \left.+\sum_{I\left(P, A^{*}, B^{*}\right) \in \mathcal{E}^{\mathrm{ext}}} \mathcal{S}_{I\left(P, A^{*}, B^{*}\right)}\right)^{\frac{1}{2}} \\
& \times\left(\sum_{I\left(P, L, A^{*}, B^{*}\right) \in \mathcal{E}^{\mathrm{int}}} \frac{2\left(R_{I\left(P, L, A^{*}, B^{*}\right)}^{\max }\right)^{2}}{\mathcal{S}_{I\left(P, L, A^{*}, B^{*}\right)}}\right. \\
& \times\left[\left(E_{P}-E_{L}\right)^{2}+\left(E_{B^{*}}-E_{A^{*}}\right)^{2}\right] \\
& \left.+\sum_{I\left(P, A^{*}, B^{*}\right) \in \mathcal{E}^{\mathrm{ext}}} \frac{\left(T_{I\left(A^{*}, B^{*}\right)}^{P}\right)^{2}}{\mathcal{S}_{I\left(P, A^{*}, B^{*}\right)}}\left(E_{P}\right)^{2}\right)^{\frac{1}{2}} .
\end{aligned}
$$

We conclude with the help of the assumption (3.35) that $\left\|E_{h}\right\|_{\mathcal{A}} \leq \widetilde{C} h$, where $\widetilde{C}$ is a strictly positive number without dependency on $h$. We summarize what precedes as
Proposition 3.9 (Error estimates) Assume that the discontinuities in $\Omega$ of the piecewise constant permeability tensor K generate a finite number of subdomains $\left\{\Omega_{s}\right\}_{s \in S}$ over which the exact solution $\varphi$ of Eqs. (1.1)-(1.2) meets the following property:

$\varphi_{\left.\right|_{\Omega_{s}}} \in C^{2}\left(\bar{\Omega}_{s}\right) \quad \forall s \in S$.

Under the conditions (1.3), (1.4), (2.12), and (2.13), the function $E_{h} \in \boldsymbol{S}(\mathcal{A})$, associated with the error vector whose components are $\left\{E_{N}=\varphi_{N}-\bar{\varphi}_{N}\right\}_{N \in \mathcal{N}}$, satisfies the following estimates:

$\left\|E_{h}\right\|_{\mathcal{A}} \leq C h \quad$ and $\quad\left\|E_{h}\right\|_{L^{2}(\Omega)} \leq C h$

where $C$ represents diverse, strictly positive, meshindependent constants.

Remark 3.10 The second inequality in Proposition 3.9 follows directly from Lemma 3.2. The first inequality ensures the convergence with order 1 (at least) of $\varphi^{\mathcal{P}}$ and $\varphi^{\mathcal{D}}$ for the norms $\|\cdot\|_{\mathcal{P}}$ and $\|\cdot\|_{\mathcal{D}}$ defined by Eqs. (3.6) and (3.7), respectively.

\subsection{Error estimates for piecewise linear approximate solutions}

It is shown in [30] for square meshes that the solution $\varphi$ to the problem (1.1)-(1.2) could be approximated with diverse continuous cellwise polynomial functions over $\bar{\Omega}$. Moreover, in [30], error estimates have been obtained for cellwise linear, bilinear, and biquadratic solutions. A generalization of these results to nonstructured meshes does not seem obvious, except for cellwise linear solution that can be obtained in a general grid context as follows. Any cell from the primary mesh $\mathcal{P}$ is a (convex) polygon and so can be split into a finite number of triangular elements (see Fig. 7). Let $\{T\}_{T \in \mathcal{T}(\mathcal{P})}$ be the triangular mesh generated by this way. So, the set of vertices of each triangular element $T \in \mathcal{T}(\mathcal{P})$ is made up of nodes where approximate

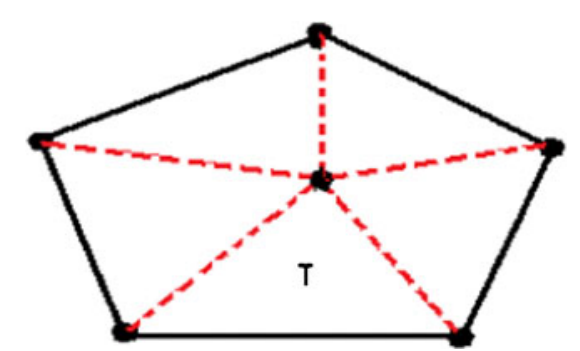

Fig. 7 Division of a primary cell into triangular elements 
pressures are available as unique solution of DDFV problem (2.40)-(2.42). Next, the exact pressure $\varphi$ is approximated in $T$ by a linear function $\Phi_{h, T}$ defined by previously computed pressures at vertices of $T$.

One can show (following [30]) that the cellwise linear approximate solution $\Phi_{h}$ associated with the triangular mesh $\{T\}_{T \in \mathcal{T}(\mathcal{P})}$ converges to the exact solution for $L^{2}$-norm. More precisely, we have

Proposition 3.11 Under the same assumptions as those of Proposition 3.9, the exact solution $\varphi$ and the piecewise linear approximate solution $\Phi_{h}$ satisfy to the following estimates:

$\left\|\wedge_{h} \varphi-\Phi_{h}\right\|_{L^{2}(\Omega)} \leq C h, \quad\left\|\wedge_{h} \varphi-\Phi_{h}\right\|_{L^{\infty}(\Omega)} \leq C h^{\frac{1}{2}}$,

$\left\|\varphi-\Phi_{h}\right\|_{L^{2}(\Omega)} \leq C h$.

where $\wedge_{h}$ represents the classical linear Lagrange interpolation operator associated with the set of nodes and where $C$ stands for diverse, strictly positive, meshindependent constants.

\section{Numerical tests and comparison with other methods}

We deal in this section with test problems extracted (with corresponding grids) from FVCA5 benchmark (see [22]). Let us start with recalling some variants of the DDFV method exposed in this work. In diverse test problems analyzed in this section, the proposed DDFV method is referred to as reused-value method.

\subsection{Variants of the proposed method}

The method exposed in this work could be slightly modified in the following way: One performs the flux approximation over each (primary and dual) cell boundary with imposing the flux continuity per halfedge. This is a variant named half-edge method in [27]. Alternatively, one could impose the flux continuity per edge for primary cell boundaries and per half-edge for dual cell boundaries. This process leads to another variant named mixed method in [27]. For more details concerning these variants, see [27].

\subsection{Notations}

We have adopted the same notations as in [22]. Indeed, we denote by nunkw the number of unknowns, nnmat the number of nonzero terms in the discrete problem matrix, and sumflux the discrete flux balance, i.e., $\operatorname{sumflux}=$ flux $0+$ flux $1+$ fluy $0+$ fluy $1-$ sumf, where flux0, flux1, fluy0, and fluy1 are respectively the outward approximate fluxes across the following boundaries: $\{(x, y) ; x=0$ and $0 \leq y \leq 1\},\{(x, y) ; x=1$ and $0 \leq y \leq 1\},\{(x, y) ; 0 \leq x \leq 1$ and $y=0\}$, and $\{(x, y) ; \quad 0 \leq x \leq 1$ and $y=1\}$ and where sumf $=$ $\sum_{P \in \mathcal{P}} \operatorname{meas}\left(C_{P}\right) f\left(x^{P}\right)$ with $x^{P}$ denoting the coordinates of the cellpoint $P$ associated with the primary cell $C_{P}$. min is the minimum value of the approximate solution while max is its maximum value. When the analytical solution is available and the mesh is refined, we set

$\operatorname{erL} 2=\left(\frac{\sum_{P \in \mathcal{P}} \operatorname{meas}\left(C_{P}\right)\left[\varphi\left(x^{P}\right)-\bar{\varphi}_{P}\right]^{2}}{\sum_{P \in \mathcal{P}} \operatorname{meas}\left(C_{P}\right)\left[\varphi\left(x^{P}\right)\right]^{2}}\right)^{\frac{1}{2}}$

which is the relative discrete $L^{2}$-norm of the error for the exact potential. Defined by analogy, ergradL2 is the relative discrete $L^{2}$-norm of the error on the exact potential gradient. We denote by erL2(i) (resp. ergradL2(i)) the relative discrete $L^{2}$-norm (resp. ergradL2) of the error on the exact potential (resp. exact potential gradient) corresponding to a level $i$ (integer $\geq 2$ ) of refinement for a given mesh. Let us set for all integers $i \geq 2$

$\operatorname{ratioL} 2(i)=-2 \frac{\ln [\operatorname{erL} 2(i)]-\ln [\operatorname{erL} 2(i-1)]}{\ln [\operatorname{nunkw}(i)]-\ln [\operatorname{nunkw}(i-1)]}$.

We define ratiogradL2(i), for all integers $i \geq 2$, with the same relation as for ratioL2(i), except that erL2 is replaced by ergradL2. We respectively denote by erflx 0 , erflx1, erfly0, and erfly1 the relative error between flx0, flx1, fly0, and fly1 and the corresponding exact flux. erflm stands for $L^{\infty}$-norm of the error on the mean value of the flux across the mesh edges. In other words, we have

$\operatorname{erflm}=\max _{\sigma \in \mathcal{E}} \frac{1}{\operatorname{meas}(\sigma)}\left|Q_{\sigma}-\bar{Q}_{\sigma}\right|$

where $Q_{\sigma}$ and $\bar{Q}_{\sigma}$ are respectively the exact and the approximate flux across $\sigma$ which is either a primary edge or a dual edge. Note that a dual edge is actually a pseudo-edge and recall that the notion of pseudo-edge is defined in Definition 2.8.

We denote by ocvL2 (resp. ocvgradL2) the order of convergence of the approximate potential (gradient of potential) in $L^{2}$-norm. ocvL2 is given by the formula

$\operatorname{ocvL} 2=\frac{\ln [\operatorname{erL} 2(\operatorname{imax})]-\ln [\operatorname{erL} 2(\operatorname{imax}-1)]}{\ln [h(\operatorname{imax})]-\ln [h(\operatorname{imax}-1)]}$

where imax is the maximum level of refinement of a given mesh and $h$ (imax) the corresponding mesh size. ocvgradL2 is defined by the same formula as for ocvL2 except that erL2 is replaced by ergradL2. ocvdisennorm 


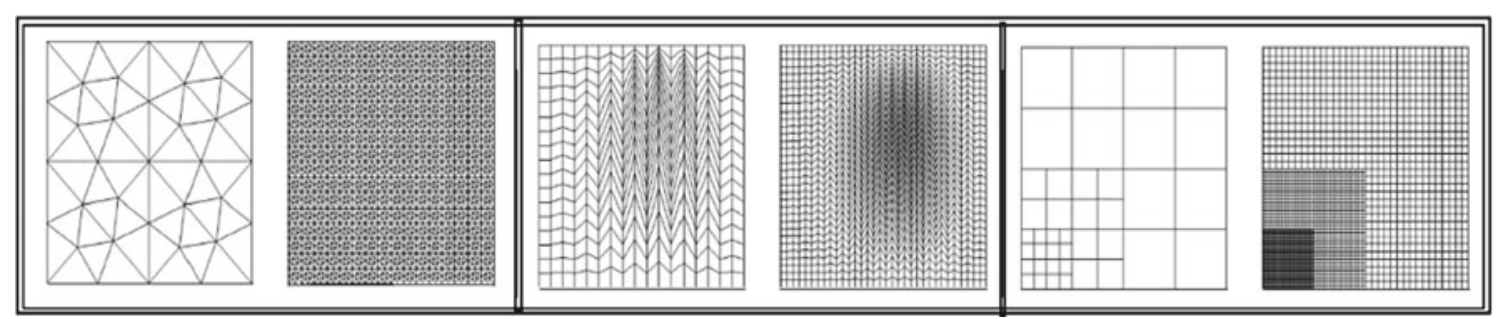

Fig. 8 Coarse and refined meshes under consideration for Part I. Left: Triangular meshes, middle: distorted quadrilateral meshes, and right: rectangular nonconforming meshes

denotes the order of convergence in the norm (3.4) of the error on the exact potential.

\subsection{Test problems}

Remark 4.1 If we had to comment on how the irregular grids are refined, we would say that irregularity is imposed on all refinement levels.

Part I: Let $\Omega$ be the square $] 0,1[\times] 0,1[$ with the following permeability tensor:

$K=\left(\begin{array}{ll}1.5 & 0.5 \\ 0.5 & 1.5\end{array}\right)$.

We equip alternatively $\Omega$ with the classes of meshes depicted in Fig. 8, which are triangular, distorted quadrilateral, and nonconforming rectangular.

- Test problem no. 1: The exact solution to Eqs. (1.1)-(1.2) is $\varphi\left(x_{1}, x_{2}\right)=16 x_{1} x_{2}\left(1-x_{1}\right)\left(1-x_{2}\right)$. Note that it is easy to get the corresponding source term $f$.

Triangular mesh solutions: For this test case, computations in Table 1 confirm our theoretical result for the reused-value method (see estimates in Proposition 3.9). On the other hand, these computations exhibit a quadratic convergence in $L^{2}$-norm for the pressure and its gradient (no contradiction with estimates from Proposition 3.9). According to the FVCA5 benchmark report (see [22]), only a few methods have achieved this level of performance. Many of them (including our contribution within that benchmark, namely the halfedge method (Table 2)) have displayed a quadratic convergence for pressure and only a linear convergence for pressure gradient in $L^{2}$-norm.

The flux errors for $L^{\infty}$-norm exhibited in Table 3 are in accordance with the theoretical results given in Proposition 3.6 which states that the flux error for $L^{\infty}$ norm is of the same magnitude order for both primal and dual edges (see also Remark 3.7).

Distorted quadrilateral mesh solutions Hereafter, $i=$ 1 is the coarse level and $i=2$ corresponds to the refined level of gridding.

According to Table 7, the quadrilateral mesh solution to the diffusion problem (1.1)-(1.2) converges much faster than the above triangular mesh one for the norm (3.4). This quadrilateral mesh solution and its gradient converge at the same rate as the triangular mesh ones for $L^{2}$-norm. The Half-edge and the mixed methods possess a common convergence rate. From the flux computation viewpoint, both of these methods are less powerful than the reused-value method (see Tables 4, 5, 6, and 7).

- Test problem no. 2: The exact solution to the diffusion problem (1.1)-(1.2) is $\varphi\left(x_{1}, x_{2}\right)=$ $\sin \left[\left(1-x_{1}\right)\left(1-x_{2}\right)\right]+\left(1-x_{1}\right)^{3}\left(1-x_{2}\right)^{2}$. Note that it is easy to determine the corresponding (source term) function $f$.

Triangular mesh solutions: In this second test prob-

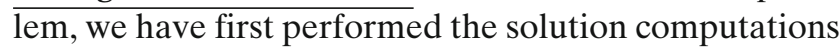

Table 1 Test problem no. 1 and triangular mesh: convergence rate of the reused-value method for $L^{2}$-norm and for discrete $H_{0}^{1}$-norm

\begin{tabular}{|c|c|c|c|c|c|c|}
\hline \multicolumn{3}{|c|}{ Parameters } & \multicolumn{4}{|c|}{ Reused-value method } \\
\hline$i$ & nunkw & nnmat & erL2 & ergradL2 & ratioL2 & ratiogradL2 \\
\hline 1 & 77 & 557 & $2.1 \mathrm{E}-02$ & $1.8 \mathrm{E}-02$ & $0.00 \mathrm{E} 00$ & $0.00 \mathrm{E} 00$ \\
\hline 2 & 321 & 2,593 & $5.8 \mathrm{E}-03$ & $5.2 \mathrm{E}-03$ & $1.79 \mathrm{E} 00$ & $1.76 \mathrm{E} 00$ \\
\hline 3 & 1,313 & 11,201 & $1.5 \mathrm{E}-03$ & $1.4 \mathrm{E}-03$ & $1.93 \mathrm{E} 00$ & $1.88 \mathrm{E} 00$ \\
\hline 4 & 5,313 & 46,561 & $3.8 \mathrm{E}-04$ & $3.7 \mathrm{E}-04$ & $1.97 \mathrm{E} 00$ & $1.89 \mathrm{E} 00$ \\
\hline 5 & 21,377 & 189,857 & $9.3 \mathrm{E}-05$ & $1.1 \mathrm{E}-04$ & $1.99 \mathrm{E} 00$ & $1.89 \mathrm{E} 00$ \\
\hline
\end{tabular}


Table 2 Test problem no. 1 and triangular mesh: convergence rate of the half-edge method for $L^{2}$-norm and for discrete $H_{0}^{1}$-norm

\begin{tabular}{|c|c|c|c|c|c|c|}
\hline \multicolumn{3}{|c|}{ Parameters } & \multicolumn{4}{|c|}{ Half-edge method } \\
\hline$i$ & nunkw & nnmat & erL2 & ergradL2 & ratioL2 & ratiogradL2 \\
\hline 1 & 77 & 557 & $2.3 \mathrm{E}-02$ & $2.7 \mathrm{E}-02$ & $0.00 \mathrm{E} 00$ & $0.00 \mathrm{E} 00$ \\
\hline 2 & 321 & 2,593 & $6.4 \mathrm{E}-03$ & $1.3 \mathrm{E}-02$ & $1.78 \mathrm{E} 00$ & $1.09 \mathrm{E} 00$ \\
\hline 3 & 1,313 & 11,201 & $1.6 \mathrm{E}-03$ & $6.2 \mathrm{E}-03$ & $1.92 \mathrm{E} 00$ & $1.04 \mathrm{E} 00$ \\
\hline 4 & 5,313 & 46,561 & $4.1 \mathrm{E}-04$ & $3.1 \mathrm{E}-03$ & $1.97 \mathrm{E} 00$ & $0.978 \mathrm{E} 00$ \\
\hline 5 & 21,377 & 189,857 & $1.0 \mathrm{E}-04$ & $1.4 \mathrm{E}-03$ & $1.98 \mathrm{E} 00$ & $0.989 \mathrm{E} 00$ \\
\hline
\end{tabular}

over triangular meshes (see Fig. 8) for the reused-value method and its two variants. Numerical results are shown in Tables 8, 9, 10, and 11.

The reused-value method displays a quadratic convergence in $L^{2}$-norm for pressure and pressure gradient, but only a linear convergence for the discrete energy norm defined by Eq. 3.4. This last result confirms our theoretical convergence result given in Proposition 3.9. The mixed method gets a less performance, while the half-edge method deceives with a poorer performance. In [22], some schemes have displayed such a poor performance for the current test problem.

Rectangular nonconforming mesh solutions. Solving the current test problem on a rectangular nonconforming mesh with the reused-value method, half-edge method, and mixed method leads to the results shown in Tables 12, 13, and 14, respectively.

Concluding remarks The convergence rates for the pressure are in concordance with the ones obtained in [22]. Concerning the pressure gradient, the reusedvalue method and the mixed method show equal performance, while the half-edge method is weaker. Note that flux errors for $L^{\infty}$-norm exhibited in Table 9 confirm our theoretical result as the numerical magnitude orders of these errors are the same for both primal and dual edges (see Proposition 3.6 and Remark 3.7).

Part II: We now consider a nonhomogeneous anisotropic porous medium $\Omega=] 0,1[\times] 0,1[$ whose perme-

Table 3 Test problem no. 1 and triangular mesh: flux error for $L^{\infty}$-norm and global flux balance with the reused-value method

Reused-value method

\begin{tabular}{llll}
\hline$i$ & $\operatorname{erflm}(i)$ & & sumflux $(i)$ \\
\cline { 2 - 3 } & Primal mesh & Dual mesh & \\
\hline 1 & $5.12 \mathrm{E}-02$ & $4.32 \mathrm{E}-02$ & $1.89 \mathrm{E}-13$ \\
2 & $4.39 \mathrm{E}-02$ & $3.48 \mathrm{E}-02$ & $1.78 \mathrm{E}-13$ \\
3 & $1.78 \mathrm{E}-02$ & $1.56 \mathrm{E}-02$ & $0.89 \mathrm{E}-13$ \\
4 & $0.85 \mathrm{E}-02$ & $0.67 \mathrm{E}-02$ & $7.12 \mathrm{E}-14$ \\
5 & $2.33 \mathrm{E}-03$ & $2.04 \mathrm{E}-03$ & $3.64 \mathrm{E}-15$ \\
\hline
\end{tabular}

ability matrix $K$ is defined as follows (see [22] and [3]):

$K\left(x_{1}, x_{2}\right)=\frac{1}{x_{1}^{2}+x_{2}^{2}}\left[\begin{array}{cc}10^{-3} x_{1}^{2}+x_{2}^{2} & \left(10^{-3}-1\right) x_{1} x_{2} \\ \left(10^{-3}-1\right) x_{1} x_{2} & x_{1}^{2}+10^{-3} x_{2}^{2}\end{array}\right]$.

- Test problem no. 3: It is easy to exhibit the unique $f$ such that the diffusion problem (1.1)(1.2) governed by the previous permeability matrix possesses as unique solution: $\varphi\left(x_{1}, x_{2}\right)=$ $\sin \left[\pi x_{1}\right] \sin \left[\pi x_{2}\right]$.

According to [22], this test case led to numerical locking difficulties for some participating schemes. We have used the same family of square meshes as the one in [22] for computing the reused-value method solutions (see Table 15). A quadratic convergence of the pressure and its gradient for $L^{2}$-norm is exhibited. Due to the homogeneous geological structure of $\Omega$ for test problems of Part I, the approximate pressure gradient and the approximate flux have the same rate of convergence. In Part II (and the next one), the situation is different as $\Omega$ is taken to be inhomogeneous; so, it is necessary to investigate the rate of convergence of approximate interface fluxes. Let Rate denote the investigated quantity (for $L^{\infty}$-norm); it could be defined as follows:

Rate $=\frac{\ln [\operatorname{erflm}(\operatorname{imax})]-\ln [\operatorname{erflm}(\operatorname{imax}-1)]}{\ln [h(\operatorname{imax})]-\ln [h(\operatorname{imax}-1)]}$

Using the relation (4.3), one can see from Table 16 that the convergence rate of the flux is almost 1.93 across the primal edges and 1.97 on the dual edges. This is a confirmation of our theoretical results which claim that the rates of convergence of the flux on primal and dual edges have the same magnitude order at least equal to 2 for $L^{\infty}$-norm (see Proposition 3.6 and Remark 3.7).

Part III: Following [22] for the test case exposed now, $\Omega=] 0,1[\times] 0,1[$ is taken to be a geologically complex porous medium (see Fig. 9). More precisely, $\Omega$ is a pile 
Table 4 Test problem no. 1 and distorted quadrilateral mesh: computation of flux error for $L^{\infty}$-norm and global flux balances with the reused-value method

\begin{tabular}{|c|c|c|c|c|c|c|c|}
\hline \multirow{3}{*}{$\begin{array}{l}\text { Level } \\
i\end{array}$} & \multicolumn{7}{|c|}{ Reused-value method } \\
\hline & \multirow[t]{2}{*}{ erflx0 } & \multirow[t]{2}{*}{ erflx1 } & \multirow[t]{2}{*}{ erfly0 } & \multirow[t]{2}{*}{ erfly1 } & \multirow[t]{2}{*}{$\operatorname{sumflux}(i)$} & \multicolumn{2}{|l|}{$\operatorname{erflm}(i)$} \\
\hline & & & & & & Primal & Dual \\
\hline 1 & $0.94 \mathrm{E}-03$ & $3.22 \mathrm{E}-04$ & $3.11 \mathrm{E}-03$ & $4.15 \mathrm{E}-03$ & $-1.20 \mathrm{E}-13$ & $2.58 \mathrm{E}-01$ & $2.74 \mathrm{E}-01$ \\
\hline 2 & $3.97 \mathrm{E}-04$ & $110 \mathrm{E}-06$ & $1.02 \mathrm{E}-04$ & $2.34 \mathrm{E}-04$ & $1.05 \mathrm{E}-14$ & $4.03 \mathrm{E}-02$ & $3.97 \mathrm{E}-02$ \\
\hline
\end{tabular}

Table 5 Test problem no. 1 and distorted quadrilateral mesh: computation of flux error for $L^{\infty}$-norm and global flux balances with the half-edge method

\begin{tabular}{llllllll}
\hline \multirow{2}{*}{\begin{tabular}{l} 
Level \\
\cline { 2 - 7 }
\end{tabular}} & \multicolumn{2}{l}{ Half-edge method } & & \\
nerflx0 & erflx1 & erfly0 & erfly1 & sumflux $(i)$ & erflm $(i)$ & Primal \\
\hline 1 & $5.09 \mathrm{E}-03$ & $2.58 \mathrm{E}-02$ & $6.11 \mathrm{E}-02$ & $1.98 \mathrm{E}-02$ & $-2.01 \mathrm{E}-12$ & $3.09 \mathrm{E}-01$ \\
2 & $2.46 \mathrm{E}-03$ & $2.25 \mathrm{E}-03$ & $1.96 \mathrm{E}-03$ & $2.75 \mathrm{E}-03$ & $1.78 \mathrm{E}-13$ & $3.52 \mathrm{E}-02$ & $2.07 \mathrm{E}-02$ \\
\hline
\end{tabular}

of anisotropic layers with a vertical fault in the middle of the structure. From the macroscopic scale viewpoint, this fault may be mathematically modeled as an interface in $\Omega$, involving discontinuities of the diffusion coefficients distributed along the line $\left\{x_{1}=\frac{1}{2}\right\}$ (see, for instance, [26] concerning details about this point of view). This discontinuity line geometrically divides $\Omega$ into two parts. Therefore, one may define $\Omega$ as a union of two anisotropic heterogenous materials, namely $\Omega_{1}$ and $\Omega_{2}$, with $\Omega_{2}=\Omega \backslash \Omega_{1}$ and $\Omega_{1}=\Omega_{1}^{l} \cup \Omega_{1}^{r}$, where

$\Omega_{1}^{l}=(0 ; .5] \times\left(\cup_{k=0}^{4}[.05+2 k \times .1 ; .05+(2 k+1) \times .1]\right)$

$\Omega_{1}^{r}=(.5 ; 1) \times\left(\cup_{k=0}^{4}[2 k \times .1 ;(2 k+1) \times .1]\right)$.
The figure below illustrates the geological structure of the anisotropic nonhomogeneous porous domain $\Omega$.

The medium permeability tensor $K$ is defined as

$$
K\left(x_{1}, x_{2}\right)=\left[\begin{array}{cc}
K_{11} & 0 \\
0 & K_{22}
\end{array}\right]
$$

with

$$
\begin{aligned}
& K_{11}=10^{2} \text { and } K_{22}=10 \text { for }\left(x_{1}, x_{2}\right) \in \Omega_{1} \\
& K_{11}=10^{-2} \text { and } K_{22}=10^{-3} \text { for }\left(x_{1}, x_{2}\right) \in \Omega_{2} .
\end{aligned}
$$

- Test problem no. 4: The pressure $\varphi$ is prescribed on the domain boundary $\Gamma$ (Dirichlet boundary conditions):

$\varphi\left(x_{1}, x_{2}\right)=1-x_{1} \quad$ on $\quad \Gamma$

\begin{tabular}{|c|c|c|c|c|c|c|c|}
\hline \multirow{3}{*}{$\begin{array}{l}\text { Level } \\
i\end{array}$} & \multicolumn{7}{|c|}{ Mixed method } \\
\hline & \multirow[t]{2}{*}{ erflx0 } & \multirow[t]{2}{*}{ erflx1 } & \multirow[t]{2}{*}{ erfly0 } & \multirow[t]{2}{*}{ erfly1 } & \multirow[t]{2}{*}{$\operatorname{sumflux}(i)$} & \multicolumn{2}{|l|}{$\operatorname{erflm}(i)$} \\
\hline & & & & & & Primal & Dual \\
\hline 1 & $2.47 \mathrm{E}-03$ & $2.06 \mathrm{E}-02$ & $2.42 \mathrm{E}-02$ & $2.11 \mathrm{E}-02$ & $0.34 \mathrm{E}-13$ & $6.85 \mathrm{E}-01$ & $\overline{5.74 \mathrm{E}-01}$ \\
\hline 2 & $1.93 \mathrm{E}-03$ & $1.91 \mathrm{E}-03$ & $1.80 \mathrm{E}-03$ & $2.04 \mathrm{E}-03$ & $2.50 \mathrm{E}-14$ & $1.77 \mathrm{E}-02$ & $0.38 \mathrm{E}-02$ \\
\hline
\end{tabular}

Table 6 Test problem no. 1 and distorted quadrilateral mesh: computation of flux error for $L^{\infty}$-norm and global flux balances with the mixed method

\begin{tabular}{|c|c|c|c|c|c|c|c|}
\hline \multicolumn{3}{|c|}{ Parameters } & \multicolumn{5}{|c|}{ Performance of reused-value method } \\
\hline$i$ & nunkw & nnmat & sumflux & erL2 & ergadL2 & ratioL2 & $\overline{\text { ratiogradL2 }}$ \\
\hline 1 & 545 & 4,641 & $-1.63 \mathrm{E}-13$ & $2.15 \mathrm{E}-02$ & $6.32 \mathrm{E}-02$ & $0.00 \mathrm{E} 00$ & $0.00 \mathrm{E} 00$ \\
\hline 2 & 2,245 & 19,669 & $-1.01 \mathrm{E}-13$ & $5.32 \mathrm{E}-03$ & $1.53 \mathrm{E}-02$ & $1.97 \mathrm{E} 00$ & 2.01E00 \\
\hline 3 & 5,101 & 45,101 & $-3.41 \mathrm{E}-13$ & $2.36 \mathrm{E}-03$ & $6.77 \mathrm{E}-03$ & $1.99 \mathrm{E} 00$ & $1.98 \mathrm{E} 00$ \\
\hline 4 & 9,113 & 80,937 & $-2.13 \mathrm{E}-13$ & $1.32 \mathrm{E}-03$ & $3.80 \mathrm{E}-03$ & $1.99 \mathrm{E} 00$ & $1.99 \mathrm{E} 00$ \\
\hline
\end{tabular}

Table 7 Test problem no. 1 and distorted quadrilateral mesh : convergence rates of the reused-value method 
Table 8 Test problem no. 2 and triangular mesh: computed rates of convergence
Table 9 Test problem no. 2 and triangular mesh: edge flux error in $L^{\infty}$-norm for the reused-value method

\begin{tabular}{|c|c|c|c|c|c|c|}
\hline \multicolumn{3}{|c|}{ Parameters } & \multicolumn{4}{|c|}{ Performance of Reused-value method } \\
\hline$i$ & nunkw & nnmat & erL2 & ergradL2 & ratioL2 & $\overline{\text { ratiogradL2 }}$ \\
\hline 1 & 77 & 557 & $3.64 \mathrm{E}-03$ & $1.17 \mathrm{E}-02$ & $0.00 \mathrm{E} 00$ & $0.00 \mathrm{E} 00$ \\
\hline 2 & 321 & 2,593 & $8.80 \mathrm{E}-04$ & $3.61 \mathrm{E}-03$ & $1.90 \mathrm{E} 00$ & $1.64 \mathrm{E} 00$ \\
\hline 3 & 1,313 & 11,201 & $2.22 \mathrm{E}-04$ & $1.05 \mathrm{E}-03$ & $1.95 \mathrm{E} 00$ & $1.76 \mathrm{E} 00$ \\
\hline 4 & 5,313 & 46,561 & $5.61 \mathrm{E}-05$ & $2.92 \mathrm{E}-04$ & $1.97 \mathrm{E} 00$ & $1.82 \mathrm{E} 00$ \\
\hline 5 & 21,377 & 189,857 & $1.41 \mathrm{E}-05$ & $8.03 \mathrm{E}-05$ & $1.99 \mathrm{E} 00$ & $1.86 \mathrm{E} 00$ \\
\hline
\end{tabular}

\begin{tabular}{|c|c|c|c|c|c|}
\hline \multicolumn{3}{|c|}{ Parameters } & \multicolumn{3}{|c|}{ Reused-value method } \\
\hline \multirow[t]{2}{*}{$\bar{i}$} & \multirow[t]{2}{*}{ nunkw } & \multirow[t]{2}{*}{ nnmat } & \multicolumn{2}{|l|}{$\operatorname{erflm}(i)$} & \multirow[t]{2}{*}{$\operatorname{sumflux}(i)$} \\
\hline & & & Primal mesh & Dual mesh & \\
\hline 1 & 77 & 557 & $5.54 \mathrm{E}-02$ & $1.21 \mathrm{E}-01$ & $3.11 \mathrm{E}-15$ \\
\hline 2 & 321 & 2,593 & $2.88 \mathrm{E}-02$ & $7.84 \mathrm{E}-02$ & $-2.53 \mathrm{E}-14$ \\
\hline 3 & 1,313 & 11,201 & $1.44 \mathrm{E}-02$ & $4.41 \mathrm{E}-02$ & $-2.22 \mathrm{E}-14$ \\
\hline 4 & 5,313 & 46,561 & $7.19 \mathrm{E}-03$ & $2.34 \mathrm{E}-02$ & $1.47 \mathrm{E}-13$ \\
\hline
\end{tabular}

Table 10 Test problem no. 2 and triangular mesh: computed rates of convergence of the half-edge method

\begin{tabular}{|c|c|c|c|c|c|c|}
\hline \multicolumn{3}{|c|}{ Parameters } & \multicolumn{4}{|c|}{ Performance of half-edge method } \\
\hline $\bar{i}$ & nunkw & nnmat & erL2 & ergradL2 & ratioL2 & ratiogradL2 \\
\hline 1 & 77 & 557 & $3.11 \mathrm{E}-03$ & $1.83 \mathrm{E}-01$ & $0.00 \mathrm{E} 00$ & $0.00 \mathrm{E} 00$ \\
\hline 2 & 321 & 2,593 & $7.88 \mathrm{E}-04$ & $1.44 \mathrm{E}-01$ & $2.05 \mathrm{E} 00$ & $3.83 \mathrm{E}-01$ \\
\hline 3 & 1,313 & 11,201 & $1.93 \mathrm{E}-04$ & $1.07 \mathrm{E}-01$ & 1.99E00 & $4.21 \mathrm{E}-01$ \\
\hline 4 & 5,313 & 46,561 & $4.79 \mathrm{E}-05$ & $7.73 \mathrm{E}-02$ & 1.99E00 & $4.62 \mathrm{E}-01$ \\
\hline 5 & 21,377 & 189,857 & $1.19 \mathrm{E}-05$ & $5.54 \mathrm{E}-05$ & $1.99 \mathrm{E} 00$ & $4.81 \mathrm{E}-01$ \\
\hline
\end{tabular}

Table 11 Test problem no. 2 and triangular mesh: computed rates of convergence of the mixed method

\begin{tabular}{|c|c|c|c|c|c|c|}
\hline \multicolumn{3}{|c|}{ Parameters } & \multicolumn{4}{|c|}{ Performance of mixed method } \\
\hline$i$ & nunkw & nnmat & erL2 & ergradL2 & ratioL2 & ratiogradL2 \\
\hline 1 & 77 & 557 & $3.26 \mathrm{E}-03$ & $1.19 \mathrm{E}-02$ & $0.00 \mathrm{E} 00$ & $0.00 \mathrm{E} 00$ \\
\hline 2 & 321 & 2,593 & $7.32 \mathrm{E}-04$ & $3.71 \mathrm{E}-03$ & 2.09E00 & $1.69 \mathrm{E} 00$ \\
\hline 3 & 1,313 & 11,201 & $1.77 \mathrm{E}-04$ & $1.15 \mathrm{E}-03$ & $2.04 \mathrm{E} 00$ & $1.65 \mathrm{E} 00$ \\
\hline 4 & 5,313 & 46,561 & 4.37E-05 & $3.95 \mathrm{E}-04$ & $2.02 \mathrm{E} 00$ & $1.65 \mathrm{E} 00$ \\
\hline 5 & 21,377 & 189,857 & $1.09 \mathrm{E}-05$ & $1.59 \mathrm{E}-04$ & $2.01 \mathrm{E} 00$ & $1.51 \mathrm{E} 00$ \\
\hline
\end{tabular}

Table 12 Test problem no. 2 and rectangular nonconforming mesh: computed convergence orders of the reused-value method

Table 13 Test problem no. 2 and rectangular nonconforming mesh: computed convergence orders

Table 14 Test problem no. 2 and rectangular nonconforming mesh: computed convergence orders

\begin{tabular}{|c|c|c|c|c|c|c|}
\hline \multicolumn{3}{|c|}{ Parameters } & \multicolumn{4}{|c|}{ Reused-value method } \\
\hline $\bar{i}$ & nunkw & nnmat & erL2 & ergradL2 & ratioL2 & $\overline{\text { ratiogradL2 }}$ \\
\hline$\overline{1}$ & 73 & 569 & $5.78 \mathrm{E}-03$ & $5.12 \mathrm{E}-02$ & $0.00 \mathrm{E} 00$ & $0.00 \mathrm{E} 00$ \\
\hline 2 & 305 & 2,561 & $1.38 \mathrm{E}-03$ & $2.00 \mathrm{E}-02$ & $1.95 \mathrm{E} 00$ & $1.31 \mathrm{E} 00$ \\
\hline 3 & 1,249 & 10,865 & $3.36 \mathrm{E}-04$ & $7.32 \mathrm{E}-03$ & 1.98E00 & $1.43 \mathrm{E} 00$ \\
\hline 4 & 5,057 & 44,753 & $8.29 \mathrm{E}-05$ & $2.6 \mathrm{E}-03$ & $1.98 \mathrm{E} 00$ & $1.48 \mathrm{E} 00$ \\
\hline \multicolumn{3}{|c|}{ Parameters } & \multicolumn{4}{|c|}{ Half-edge method } \\
\hline$i$ & nunkw & nnmat & erL2 & ergradL2 & ratioL2 & $\overline{\text { ratiogradL2 }}$ \\
\hline 1 & 73 & 569 & $5.44 \mathrm{E}-03$ & $1.94 \mathrm{E}-01$ & $0.00 \mathrm{E} 00$ & $0.00 \mathrm{E} 00$ \\
\hline 2 & 305 & 2,561 & $1.27 \mathrm{E}-03$ & $1.33 \mathrm{E}-01$ & 2.03E00 & $5.38 \mathrm{E}-01$ \\
\hline 3 & 1,249 & 10,865 & $3.15 \mathrm{E}-04$ & $9.11 \mathrm{E}-02$ & $1.98 \mathrm{E} 00$ & $5.32 \mathrm{E}-01$ \\
\hline 4 & 5,057 & 44,753 & $7.86 \mathrm{E}-05$ & $6.28 \mathrm{E}-02$ & $1.98 \mathrm{E} 00$ & $5.07 \mathrm{E}-01$ \\
\hline \multicolumn{3}{|c|}{ Parameters } & \multicolumn{4}{|c|}{ Mixed method } \\
\hline $\bar{i}$ & nunkw & $\overline{\text { nnmat }}$ & erL2 & ergradL2 & ratioL2 & $\overline{\text { ratiogradL2 }}$ \\
\hline 1 & 73 & 569 & $5.62 \mathrm{E}-03$ & $4.86 \mathrm{E}-02$ & $0.00 \mathrm{E} 00$ & $0.00 \mathrm{E} 00$ \\
\hline 2 & 305 & 2,561 & $1.32 \mathrm{E}-03$ & $1.88 \mathrm{E}-02$ & 2.03E00 & $1.33 \mathrm{E} 00$ \\
\hline 3 & 1,249 & 10,865 & $3.24 \mathrm{E}-04$ & $6.91 \mathrm{E}-03$ & $2.01 \mathrm{E} 00$ & $1.42 \mathrm{E} 00$ \\
\hline 4 & 5,057 & 44,753 & $7.98 \mathrm{E}-05$ & $2.47 \mathrm{E}-03$ & $2.00 \mathrm{E} 00$ & $1.47 \mathrm{E} 00$ \\
\hline
\end{tabular}


Table 15 Test problem no. 3: convergence orders for the reused-value method

\begin{tabular}{|c|c|c|c|c|c|c|}
\hline \multicolumn{3}{|c|}{ Parameters } & \multicolumn{4}{|c|}{ Computed convergence orders } \\
\hline$i$ & nunkw & nnmat & erL2 & ergradL2 & ratioL2 & ratiogradL2 \\
\hline 1 & 25 & 169 & $1.14 \mathrm{E}-02$ & $6.68 \mathrm{E}-02$ & 00E00 & 00E00 \\
\hline 2 & 113 & 897 & $2.41 \mathrm{E}-03$ & $7.28 \mathrm{E}-03$ & $1.88 \mathrm{E} 00$ & $1.73 \mathrm{E} 00$ \\
\hline 3 & 481 & 4,081 & $5.88 \mathrm{E}-04$ & $1.87 \mathrm{E}-03$ & $1.94 \mathrm{E} 00$ & $1.89 \mathrm{E} 00$ \\
\hline 4 & 1,985 & 17,361 & $1.40 \mathrm{E}-04$ & $4.78 \mathrm{E}-04$ & $1.99 \mathrm{E} 00 \mathrm{E}$ & $1.96 \mathrm{E} 00$ \\
\hline 5 & 8,065 & 71,569 & $3.48 \mathrm{E}-05$ & $1.19 \mathrm{E}-04$ & $2.00 \mathrm{E} 00 \mathrm{E}$ & $1.99 \mathrm{E} 00$ \\
\hline
\end{tabular}

Table 16 Test problem no. 3: flux error in $L^{\infty}$-norm of the reused-value method for primal and dual edges

\begin{tabular}{|c|c|c|c|c|c|}
\hline \multicolumn{3}{|c|}{ Parameters } & \multicolumn{3}{|c|}{ Reused-value method } \\
\hline \multirow[t]{2}{*}{$\bar{i}$} & \multirow[t]{2}{*}{ nunkw } & \multirow[t]{2}{*}{$h(i)$} & \multicolumn{2}{|c|}{$\operatorname{erflm}(i)$} & \multirow[t]{2}{*}{$\operatorname{sumflux}(i)$} \\
\hline & & & Primal mesh & Dual mesh & \\
\hline 1 & 25 & $1 / 4$ & $3.25 \mathrm{E}-01$ & $2.87 \mathrm{E}-01$ & $1.78 \mathrm{E}-15$ \\
\hline 2 & 113 & $1 / 8$ & $2.49 \mathrm{E}-01$ & $1.53 \mathrm{E}-01$ & $4.44 \mathrm{E}-15$ \\
\hline 3 & 481 & $1 / 16$ & $1.43 \mathrm{E}-01$ & $7.72 \mathrm{E}-02$ & $1.15 \mathrm{E}-14$ \\
\hline 4 & 1,985 & $1 / 32$ & $7.55 \mathrm{E}-02$ & $3.99 \mathrm{E}-02$ & $1.27 \mathrm{E}-13$ \\
\hline 5 & 8,065 & $1 / 64$ & $1.98 \mathrm{E}-02$ & $1.02 \mathrm{E}-02$ & $0.63 \mathrm{E}-14$ \\
\hline
\end{tabular}

and the source term $f$ is taken to be zero. According to the (continuous) maximum principle, the exact solution for the system (1.1)-(1.2) should be bounded by 0 and 1 . Our purpose in this test case is to check if the computed solution takes its values between 0 and 1. For this purpose, a rectangular coarse mesh (see Fig. 10) and a refined conforming square mesh (made up of $20 \times 20$ cells) have been considered.

We are also interested in (1) knowing whether the proposed method meets the global mass conservation in $\Omega$ and (2) computing the rate of convergence for the approximate interface fluxes. Since the global energy dissipated (in $\Omega$ by viscous forces related to the flow) could be used to compute the effective permeability tensor of $\Omega$ (see, for instance, [29, 35] but also [12] and the references therein), we are interested in in-

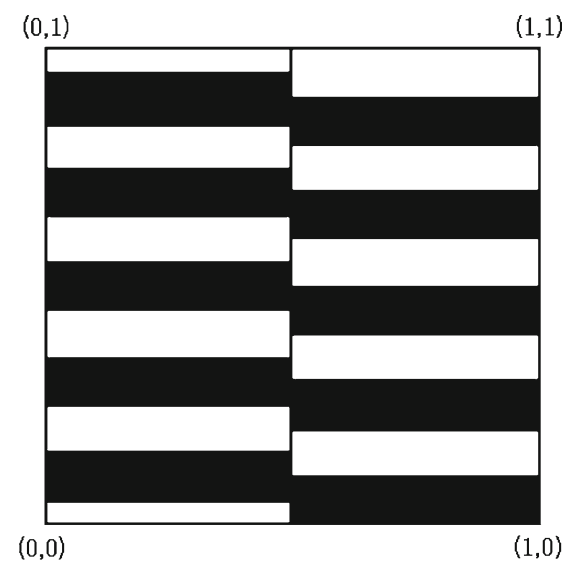

Fig. 9 Geological architecture of the porous medium $\Omega$ vestigating the capabilities of the reused-value method to compute this energy (whose exact value is almost $43.2 E 00$ and could be obtained from a reference square mesh made of $320 \times 320$ cells).

Let us start with some definitions and notations (inspired from [22]). We denote by Ener1 and Ener2 two approximations of the same quantity (namely the global energy dissipated in $\Omega$ ) based on two integral expressions (perfectly identical in their continuous setting):

$$
\begin{aligned}
& E_{1}=\int_{\Omega}(K \operatorname{grad} \varphi) \cdot \operatorname{grad} \varphi d x, \\
& E_{2}=\int_{\Gamma} \varphi(K \operatorname{grad} \varphi \cdot n) d s
\end{aligned}
$$

$(0,1)$

$(1,1)$

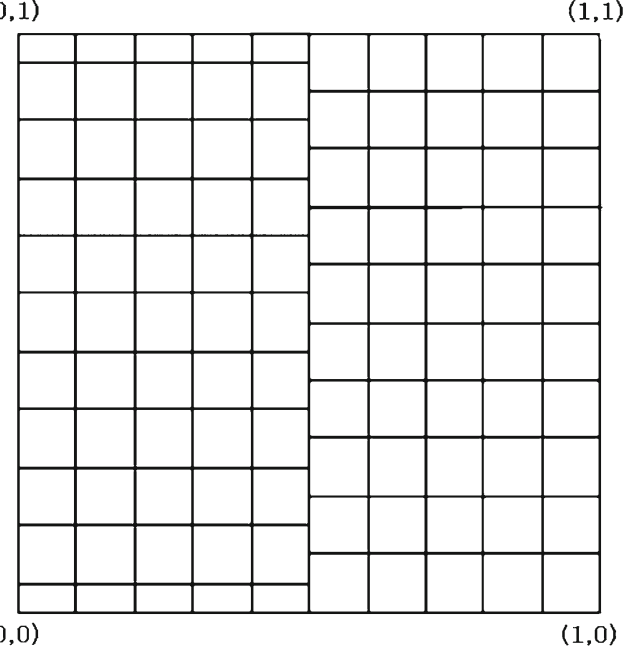

Fig. 10 A nonconforming rectangular coarse mesh defined on $\Omega$ 
Table 17 Test problem no. 4 with discontinuous permeability tensor and a vertical fault in $\Omega$ : flux error in $L^{\infty}$-norm of the reused-value method for primal and dual edges

\begin{tabular}{|c|c|c|c|c|}
\hline \multicolumn{2}{|l|}{ Parameters } & \multicolumn{3}{|c|}{ Reused-value method and flux computation } \\
\hline \multirow[t]{2}{*}{$i$} & \multirow[t]{2}{*}{$h(i)$} & \multicolumn{2}{|l|}{$\operatorname{erflm}(i)$} & \multirow[t]{2}{*}{$\operatorname{sumflux}(i)$} \\
\hline & & Primal mesh & Dual mesh & \\
\hline Coarse $=1$ & $1 / 10$ & $1.26 \mathrm{E}-01$ & $1.67 \mathrm{E}-01$ & $6.85 \mathrm{E}-11$ \\
\hline Refined $=2$ & $1 / 20$ & $3.25 \mathrm{E}-02$ & $4.19 \mathrm{E}-02$ & $9.07 \mathrm{E}-13$ \\
\hline
\end{tabular}

Table 18 Test problem no. 4 with discontinuous permeability tensor and a vertical fault in $\Omega$ : verification of the maximum principle and computation of viscous force energy with formulas shown in Eq. (4.9)

\begin{tabular}{|c|c|c|c|c|c|c|c|}
\hline \multicolumn{3}{|c|}{ Parameters } & \multicolumn{5}{|c|}{ Reused-value method and energy computation } \\
\hline Level & nunkw & nnmat & Min & Max & Ener1 & Ener2 & Eren \\
\hline Coarse & 200 & 1,291 & $-1.66 \mathrm{E} 00$ & 2.29E00 & $6.72 \mathrm{E} 01$ & 4.45E01 & $3.38 \mathrm{E}-01$ \\
\hline Refined & 761 & 4,507 & $0.00 \mathrm{E} 00$ & $1.00 \mathrm{E} 00$ & 4.36E01 & 4.34E01 & $4.6 \mathrm{E}-03$ \\
\hline
\end{tabular}

The relative error Eren is defined by relation (4.10)

On the other hand, Eren denotes the quantity defined by the relation

Eren $=\frac{\mid \text { Ener1 }- \text { Ener2 } \mid}{\max \{\text { Ener1, Ener2 }\}}$.

Thanks to relation (4.3), one can see from Table 17 that the convergence rate of the flux is 1.95 across the primal edges and grows into 1.99 on the dual edges. So, the flux globally converges slightly faster on dual edges than it does on primal edges. These convergence rates confirm our theoretical results that assert a convergence of order 2 (at least) for edge fluxes in $L^{\infty}$ norm. On the other hand, it is clear from Table 18 that if the mesh becomes finer and finer, the DDFV approximation of the energy expressions given by Eq. (4.9) leads to almost the same real value that converges to the energy dissipated by viscous forces in $\Omega$.

\section{Conclusions and perspectives}

In this work, we have presented a theoretical analysis and a numerical implementation of the DDFV method for subsurface flow problems. The discrete solutions are nothing than approximate pressures at cellpoints and vertices. With any discrete solution, a $P_{1}$-finite volume solution is associated. We have proven the stability and some error estimates for the discrete solution (see Proposition 3.5 and Proposition 3.9). From these error estimates, the convergence of a $P_{1}$-finite volume solution with order 1.00 for $L^{2}$-norm and 0.50 for $L^{\infty}$-norm is derived. From the implementation and comparison point of view, the efficiency and competitiveness of the reused-value method have been shown for diverse unstructured and/or nonconforming meshes. Especially, convergence (with the same magnitude order) of approximate fluxes across primal and dual edges is theoretically and computationally proven. The theoretical analysis of this method for nonconforming unstructured meshes could be an interesting challenge.

Acknowledgements The authors would like to greatly thank the anonymous referees for their constructive remarks and useful suggestions that have contributed to significantly improve many parts of this work. The authors would also like to express their gratitude to the Ministry of Higher Education of Cameroon for its financial support to this work.

Open Access This article is distributed under the terms of the Creative Commons Attribution License which permits any use, distribution, and reproduction in any medium, provided the original author(s) and the source are credited.

\section{References}

1. Aavatsmark, I.: Multi-point flux approximation methods for quadrilateral grids. In: 9th International Forum on Reservoir Simulation, Abu Dhabi (2007)

2. Aavatsmark, I., Barkve, T., BOE, O., Mannseth, T.: Discretization on non-orthogonal curvilinear grids for multi-phase flow. In: Proceedings of the 4th ECMOR, Roros, Norway (1994)

3. Andreianov, B., Boyer, F., Hubert, F.: Discrete duality finite volume schemes for Leray-Lions-type elliptic problems on general 2D meshes. Numer. Methods Partial Differ. Equ. 23(1), 145-195 (2007)

4. Andreianov, B., Gutnic, M., Wittbld, P.: Convergence of finite volume approximations for a nonlinear elliptic parabolic problem: a continuous approach. SIAM J. Numer. Anal. 42(1), 228-251 (2004)

5. Arbogast, T., Wheeler, M., Yotov, I.: Mixed finite elements for elliptic problems with tensor coefficients as cell-centered finite differences. SIAM J. Numer. Anal. 34, 828-852 (1997)

6. Berndt, M., Lipnikov, K., Shashkov, M., Wheeler, M.F., Yotov, I.: Superconvergence of the velocity in mimetic finite difference methods on quadrilaterals. SIAM J. Numer. Anal. 43, 1728-1749 (2005) 
7. Brezzi, F., Douglas, J., Marini, L.D.: Two families of mixed finite elements for second order elliptic problems. Numer. Math. 47, 217-235 (1985)

8. Brezzi, F., Lipnikov, K., Shashkov, M.: Convergence of mimetic finite difference method for diffusion problems on polyhedral meshes with curved faces. Math. Models Methods Appl. Sci. 16, 275-297 (2006)

9. Boyer, F., Hubert, F.: Finite volume method for 2D linear and non-linear elliptic problems with discontinuities. SIAM J. Numer. Anal. 46(6), 3032-3070 (2008)

10. Chou, S.H., Kwak, D.Y., Kim, K.Y.: A general framework for constructing and analyzing mixed finite volume methods on quadrilateral grids: the overlapping covolume case. SIAM J. Numer. Anal. 39(4), 1170-1196 (2001)

11. Chen, Q.Y., Wan, J., Yang, Y., Mifflin, R.T.: Enriched multipoint flux approximation for general grids. J. Comput. Phys. 227(3), 1701-1721(2008)

12. Durlofsky, L.J.: Upscaling and gridding of geologically complex systems. Department of Petroleum Engineering, Stanford University Chevron Texaco E \& P Technology Company (2005)

13. Domelevo, K., Omnes, P.: A finite volume method for the Laplace equation on almost arbitrary two-dimensional grids. ESAIM Math. Model. Numer. Anal. 39(6), 1203-1249 (2005)

14. Eymard, R., Gallouet, T., Herbin, R.: Finite volume methods. In: Ciarlet, P.G., Lions, J.L. (eds.) Handbook of Numerical Analysis, VII, pp. 713-1020. North-Holland, Amsterdam (2000)

15. Eymard, R., Gallouet, T., and Herbin, R.: A cell-centered finite volume approximation, for anisotropic diffusion operators on unstructured meshes in any space dimension. IMA J. Numer. Anal. 26(2), 326-353 (2006)

16. Eymard, R., Gallouet, T., and Herbin, R.: A new finite volume scheme for anisotropic diffusion problems on general grids: convergence analysis. C.R. Math., Acad. Sci. Paris 344(6), 40340 (2007)

17. Eymard, R., Gallouet, T., Herbin, R.: Discretization schemes for linear diffusion operators on general non-conforming meshes. In: Eymard, R., Herard, J.M. (eds.) Proceedings of FVCA5, pp. 375-382. Wiley, New York (2008)

18. Eymard, R., Gallouet, T., Herbin, R.: Discretization of heterogeneous and anisotropic diffusion problems on general nonconforming meshes. SUSHI: a scheme using stabilisation and hybrid interfaces. IMA J. Numer. Anal. 30(4), 1009-1043 (2010)

19. Hermeline, F.: A finite volume method for approximating 3D diffusion operators on general meshes. J. Comput. Phys. 228(17), 5763-5786 (2009)

20. Hermeline, F.: Approximation of $2 \mathrm{D}$ and $3 \mathrm{D}$ diffusion operators with variable full tensor coefficients on arbitrary meshes. Comput. Methods Appl. Mech. Eng. 196(1), 24972526 (2007)

21. Hermeline, F.: Approximation of diffusion operators with discontinuous tensor coefficients on distorted meshes. Comput. Methods Appl. Mech. Eng. 192(16-18), 1939-1959 (2003)

22. Herbin, R., Hubert, F.: Benchmark on discretization schemes for anisotropic diffusion problems on general grids. In: Eymard, R., Herard, J.M. (eds.) Finite Volume for Complex Applications V, pp. 659-692. Wiley, London (2008)

23. Klausen, R.A., Radu, F.A., Eigestad, G.T.: Convergence of MPFA on triangulations and for Richard's equation. Int. J. Numer. Methods Fluids 58(12), 1327-1351 (2008)
24. Lazarov, R.D., Vassilevski, P.S.: Numerical methods for convection-diffusion problems on general grids. In: Bojanov, B. (ed.) Proceedings of an International Conference on "Approximation Theory", pp. 258-283. Sofia (2002)

25. Mishev, I.D.: Nonconforming finite volume methods. Comput. Geosci. 6, 253-268 (2002)

26. Martin, V., Jaffré, J., Roberts, J.E.: Modeling fractures and barriers as interfaces for flow in porous media. SIAM J. Sci. Comput. 26(5), 1667-1691 (2005)

27. Moukouop-Nguena, I., Njifenjou, A.: A new finite volume formulation for diffusion problems in anisotropic nonhomogeneous media. In: Proceedings of the 4th International Symposium on Finite Volume for Complex Applications 4, pp. 435-446. Morocco (2005)

28. Moukouop-Nguena, I., Njifenjou, A.: Benchmark on discretization schemes for anisotropic problems on general grids: some MPFA methods of DDFV type. In: Eymard, R., Herard, J.M. (eds.) Proceedings of the International Conference on "Finite Volume for Complex Applications V". Wiley, London (2008)

29. Njifenjou, A.: Expression en termes d'énergie pour la perméabilité absolue effective. Application au calcul numérique d'écoulement en milieu poreux. Oil Gas Sci. Technol. 49(4), 345-358 (1994) (Review of Institut Francais du Pétrole)

30. Njifenjou, A., Kinfack, A.J.: Convergence analysis of an MPFA method for flow problems in anisotropic heterogeneous porous media. Int. J. Finite Vol. 5(1), 17-56 (2008) (published online)

31. Njifenjou, A., Moukouop-Nguena, I.: Traitement des anisotropies de perméabilité en simulation d'écoulement en milieu poreux par les volumes finis. In: Tchuente, M. (ed.) Proceedings of an International Conference on "Systèmes Informatiques pour la Gestion de l'Environnement", pp. 245-259. Douala, Cameroon (2001)

32. Njifenjou, A., Moukouop-Nguena, I.: A finite volume approximation for second order elliptic problems with a full matrix on quadrilateral grids: derivation of the scheme and a theoretical analysis. Int. J. Finite Vol. 3(2), 64-93 (2006) (electronic publication)

33. Omnes, P.: Error estimates for a finite volume method for the Laplace equation in dimension one through discrete Green functions. Int. J. Finite Vol. 6(1), 24-41 (2009) (online publication)

34. Omnes, P.: On the second-order convergence of a function reconstructed from finite volume approximations of the Laplace equation on Delaunay-Voronoi meshes. ESAIM: Mathematical Modelling and Numerical Analysis 45( $\left.\mathrm{N}^{\circ} 04\right)$, 627-650 (2011)

35. Renard, P.: Averaging methods for permeability fields, development, protection, management and sequestration of subsurface. Fluid Flow and Transport in Porous and Fractured Media. Summer School, Cargèse, France (2005)

36. Raviart, P.A., Thomas, J.M.: A mixed finite element method for 2nd order elliptic problems. In: Dold-Eckmann (ed.) Lecture Notes in Mathematics, vol. 606, pp. 292-315. Springer, Berlin (1977)

37. Rogers, M.G., Rogers, C.F.: A flux continuous scheme for the full tensor pressure equation. In: Proceedings of the Fourth ECMOR, Roros, Norway (1994)

38. Russel, T.F., Wheeler, M.F., Yotov, I.: Superconvergence for control-volume mixed finite element methods on rectangular grids. SIAM J. Numer. Anal. 45(1), 223-235 (2007) 